Pacific

Journal of

Mathematics

CURVES ON NORMAL RATIONAL CUBIC SURFACES

JOHN BREVIK

Volume $230 \quad$ No. 2

April 2007 


\title{
CURVES ON NORMAL RATIONAL CUBIC SURFACES
}

\author{
JOHN BREVIK
}

\begin{abstract}
Let $k$ be an algebraically closed field, let $X_{0}$ be a rational normal cubic surface in $\mathbb{P}^{3}=\mathbb{P}_{k}^{3}$, and let $C_{0} \subset X_{0}$ be a locally Cohen-Macaulay curve, which is therefore an effective Weil divisor on $X_{0}$. I show that $C_{0}$ can be expressed as the limit of a family of curves whose general member lies on a smooth surface, in the following sense: There exists a flat family $X_{t}$ of cubic surfaces specializing to $X_{0}$ and a flat family $C_{t}$ of curves specializing to $C_{0}$, parametrized by a smooth (noncomplete) curve $T$, such that the general member of $X_{t}$ is a smooth cubic surface and $C_{t} \subset X_{t}$ is an effective (Cartier) divisor for all $t \in T \backslash\{0\}$.
\end{abstract}

\section{Introduction}

Let $k$ be an algebraically closed field. In this paper, I will use the term curve to refer to a locally Cohen-Macaulay scheme of pure dimension 1 over $k$; a space curve is such a curve embedded as a subscheme of $\mathbb{P}^{3}=\mathbb{P}_{k}^{3}$. As has been frequently observed, even if one's primary interest is in smooth space curves, one needs to work in the more general category of space curves as defined above in order to use the modern ideas of linkage and minimal curves, since this category is the closure of the category of smooth space curves under liaison (see, for example of [Hartshorne 1980, Section 4] for a discussion of this point).

A common approach to studying space curves is to study the curves on a particular surface or class of surfaces. For example, Gruson and Peskine [1982] constructed a quartic surface with a double line and showed that for any degree-genus pair in a certain range there is a smooth curve with this degree and genus on this quartic, thus answering the question which $(d, g)$ are possible for smooth space curves. Phrased in terms of Hilbert schemes, any $H_{g}^{d}$ which contains a smooth curve contains a curve lying on a plane, a smooth quadric surface, a smooth cubic surface, or the quartic with a double line. Mori [1984] then showed that in fact any $(d, g)$ pair that occurs for a smooth curve on this quartic also occurs for a smooth curve on a smooth quartic; this surface, however, depends on the pair $(d, g)$.

MSC2000: primary 14H50; secondary $14 \mathrm{~J} 17$.

Keywords: classification of space curves, curves on singular surfaces, singular del Pezzo surfaces. 
There is also the finer question of which irreducible components of the Hilbert scheme have curves on a given surface or on a member of a class of surfaces. There is little work in the literature in this direction, but one can ask a number of interesting questions along these lines:

(1) For a given curve $C$ on a given singular surface $S$ of degree $s$, is it true that, in the Hilbert scheme, $C$ is in the closure of the space of curves on smooth surfaces of degree $s$ ?

(2) For a given singular surface $S$, is it true that all curves on $S$ are in the closure of the set of curves on smooth surfaces as above?

(3) If one stratifies in some way the space of all surfaces of a given degree, surfaces in which strata contain the most general curves in some component of some Hilbert scheme?

Here I investigate these questions for $S$ a rational normal surface of degree 3. That the questions are not trivial is shown by a few examples. Consider:

Theorem [Hartshorne 1997]. Let $S_{0} \subset \mathbb{P}^{3}$ be a quadric cone. There exists a family of smooth quadric surfaces $S_{t}$ parametrized by a smooth curve $T$ and specializing to $S_{0}$ such that every effective Weil divisor $C_{0}$ on $S_{0}$ is the limit of a flat family of curves $C_{t}$, where $C_{t} \subset S_{t}$ for all $t \in T$.

Thus, at least some singular surfaces satisfy the very strong property (2) above.

On the other hand, if one allows a quadric surface to degenerate into the union of two planes, there are many curves on this union whose $(d, g)$ pairs do not correspond to the values which arise from curves on a smooth quadric, so certainly not every surface satisfies property (2). Even if we insist that our special surface be integral, it may not satisfy this property: Gruson and Peskine [1982] have produced an example of a family of curves of degree 13 and genus 18, lying on a cubic scroll with a double line, for which they show by dimension-counting that the general member cannot be the specialization of a flat family of curves on smooth cubic surfaces. In [Brevik and Mordasini 2003] we gave very strong necessary conditions for a curve to be such a specialization; a consequence of these conditions is a generalization of Gruson and Peskine's example: In fact, no smooth curve of degree greater than 10 on this surface is the specialization of a flat family of curves on smooth cubic surfaces.

The present work answers the question (2) above (and therefore question (1) as well) in the affirmative for curves on rational normal cubic surfaces in $\mathbb{P}^{3}$. In fact, the result is stronger, since it shows that the necessary deformation of surfaces is independent of the curve chosen.

Theorem. Let $X_{0} \subset \mathbb{P}^{3}$ be a rational normal cubic surface. Then there exists a (not necessarily complete) curve $T$ with point 0 and a flat family $X \rightarrow T$ of cubic 
surfaces in $\mathbb{P}^{3}$ such that $X_{0}$ is the fibre over $0, X_{t}$ is smooth for $t \neq 0$, and such that any effective Weil divisor $C_{0}$ on $X_{0}$ is the limit of a flat family of curves $C$ over $T$ with $C_{t} \subset X_{t}$ for each $t \in T$.

With respect to question (3) above, then, the most general member of an irreducible component of a Hilbert scheme is never contained in a rational normal cubic surface and not a smooth cubic. This result is again a generalization of the work of Gruson and Peskine, in the following sense: In the proof of [Gruson and Peskine 1982, Théorème 2.11], root systems are used to show that any $(d, g)$ pair for a smooth curve on a rational normal cubic surface already occurs as a $(d, g)$ pair for a smooth curve on a smooth cubic surface. The method of proof and the current paper's elementary Proposition 5.19 together imply the result for smooth curves (at least in characteristic 0). The difficulty, then, is to construct an appropriate flat family for general curves in our sense, which may be reducible or nonreduced.

It is natural to ask whether this result generalizes to surfaces of higher degree. Consider, however, the example of a quartic surface $S$ with one $\mathbf{A}_{1}$ singularity and a line $L$ containing the singular point. Then it is easy to show that the Weil divisor $2 L$ on $S$ has arithmetic genus -2 , and there simply are no curves on a smooth quartic of degree 2 and genus -2 , since the arithmetic genus of a double line on a smooth quartic is always -3 . On the other hand, Proposition 5.5 below shows that, for any rational double point surface singularity in $\mathbb{P}^{3}$, the problem of lifting a divisor to a smooth surface is at least "locally unobstructed," in some sense, relative to the combinatorics of the exceptional curves on the desingularization. I believe then, that it is natural to conjecture that, given a surface of degree $d$ in $\mathbb{P}^{3}$ whose only singularities are rational double points, any sufficiently positive (maybe numerically effective) divisor can be expressed as the flat limit of a family whose general member lies on a smooth surface of degree $d$.

Section 1 of this paper briefly recalls some relevant facts about ideals defining 0 -dimensional subschemes of a smooth surface and establishes the terminology regarding these objects that will be used in this paper. Section 2 concerns the construction, from the standpoint of blowing up the plane, of a normal cubic surface in $\mathbb{P}^{3}$. Section 3 "relativizes" the blowing-up process; this technique is used in Section 4 to construct families of surfaces. Section 5 consists largely of a study of numerical invariants of curves on cubic surfaces and culminates in the proof of the main theorem.

\section{Complete ideals and base loci}

This material can be found in [Lipman 1969; 1988]; what it does for us is to establish a dictionary between base conditions on linear systems on a smooth surface and 
0-dimensional subschemes of that surface. For a thorough treatment, specialized to our particular situation, see [O'Sullivan 1996].

For any ideal $\mathscr{I}$ defining a 0 -dimensional subscheme of a smooth surface $Y$, and for any point $P$ on $Y$, define $\operatorname{ord}_{P}(\mathscr{I})$ to be the largest power of the maximal ideal $\mathfrak{m}_{P}$ in the local ring $\mathscr{O}_{P}$ which contains $\mathscr{I}_{P}$. Also, if $X \rightarrow Y$ is obtained by blowing up a finite sequence of closed points, define the transform $\Phi^{X}$ of $\mathscr{I}$ on $X$ to be the ideal obtained from $\Phi_{O_{Y}}$ by factoring out the invertible part, so that $\Phi^{X}$ again defines a 0 -dimensional subscheme. For any point $Q$ existing on some blown-up surface above $Y$, define $\operatorname{ord}_{Q}(\mathscr{Y})$ to be $\operatorname{ord}_{Q}\left(\Phi^{X}\right)$, where $X$ is the minimal surface above $Y$ for which $Q$ exists as an ordinary point. Then $\mathscr{I}$ gives rise to a formal sum of points $\sum_{Q} \operatorname{ord}_{Q}(\mathscr{I}) \cdot Q$, to which I will refer as the base locus of $\mathscr{I}$. (In [Lipman 1988], this is called the point basis of $\mathscr{I}$.)

Recall that if $I$ is an ideal of a ring $R$, an element $r$ of $R$ is integral over $I$ if $r$ satisfies a polynomial

$$
r^{n}+a_{1} r^{n-1}+\cdots+a_{n},
$$

where each $a_{i} \in I^{i}$. The completion of $I$ is the set of elements of $R$ which are integral over $I$; we say that $I$ is complete if it contains all elements of $R$ integral over $I$. One can show that the completion of $I$ is a complete ideal, and also that completeness is a local property, so it makes sense to speak of the completion of an ideal sheaf.

Proposition 1.1 [Lipman 1988, Proposition 1.10]. Let $Y$ be a smooth surface. Two ideals $\mathscr{I}, \mathscr{I}$ of finite colength on $Y$ have the same base locus if and only if they have the same completion.

Theorem 1.2 [Lipman 1988, Theorem 3.1]. Let $Y$ be a smooth surface, and let $\Phi$ be a complete ideal sheaf of $Y$ defining a 0 -dimensional subscheme $Z$. Then the base locus $\sum_{Q} \operatorname{ord}_{Q}(\mathscr{I}) \cdot Q$ of $\Phi$ is a finite sum, and the length of $Z$ is equal to

$$
\sum_{Q} \frac{1}{2}\left(\operatorname{ord}_{Q}(\mathscr{I})\right)\left(\operatorname{ord}_{Q}(\mathscr{I})+1\right) .
$$

Remark 1.2.1. Thus, the base locus defines a mapping from the set of ideals on $Y$ of finite colength to the free additive monoid on the set of (equivalence classes of) points on surfaces birationally dominating $Y$. The fibres are ideals having the same completion. The image is the set of point sums satisfying the proximity inequalities (compare [Zariski 1971, Chapter II, §2]). The proximity inequalities are the set of conditions that no point in a sum has a coefficient which is smaller than the sum of the coefficients of the points infinitely near to it.

Therefore, there is a one-to-one correspondence between the set of complete ideals of finite colength on a surface $Y$ and the set of formal sums of points on surfaces birationally dominating $Y$ which satisfy the proximity inequalities. 
Proposition 1.3 [Lipman 1988, Lemma 1.11]. Suppose that $Z$ is a 0-dimensional scheme on the smooth projective surface $Y$ with base locus $B$. Let $P$ be a closed point on $Y$, and let $Y_{P} \stackrel{\pi}{\rightarrow} Y$ be the blowing-up of $Y$ at $P$, with exceptional curve $E$. Then $\Phi_{Z} \cdot \mathcal{O}_{Y_{P}}=\mathscr{I}_{E}^{\operatorname{ord}_{P}(Z)} \cdot \mathscr{I}_{Z^{\prime}}$, where $Z^{\prime}$ is a 0 -dimensional subscheme of $Y_{P}$. Then the base locus $B^{\prime}$ of $Z^{\prime}$ obeys the formula

$$
B=B^{\prime}+\operatorname{ord}_{P}(Z) \cdot P,
$$

where we identify infinitely near points on the two surfaces in the obvious way.

As shorthand, I will sometimes refer to a sum of points on $\mathbb{P}^{2}$ which satisfy the proximity inequalities as a base locus without specifying its ideal. I will refer to a sum of points $P_{1}+\cdots+P_{n}$ such that, for $i=2, \ldots, n, P_{i}$ is infinitely near to $P_{i-1}$ and to no other $P_{j}$ as a tower.

\section{Blowing up $\mathbb{P}^{2}$ at six points}

In this section, we will establish some preliminaries for studying rational normal cubic surfaces in $\mathbb{P}^{3}$. The point of view is that such a surface arises from the blowing-up of a set of 6 points in $\mathbb{P}^{2}$, with some of the points possibly infinitely near, satisfying generality conditions (Proposition 2.4). In fact, with the exception of the final result (Theorem 2.5), all of the results in this section are easy to establish using means analogous to those for smooth cubics (see, for example, [Hartshorne 1977, V, Section 4]).

Definition 2.1. Let $V$ be a smooth quasiprojective surface (over $k$ ) and let $\widetilde{V} \rightarrow V$ be a finite composition of blowings-up of closed points $\left(Q_{1}, \ldots, Q_{n}\right)$. The strict exceptional divisor $E_{i}$ on $\widetilde{V}$ is defined to be the strict transform of the exceptional curve obtained when $Q_{i}$ is blown up. The total exceptional divisor $e_{i}$ is the total transform of the point $Q_{i}$.

Remark 2.1.1. In case the sequence $\left(Q_{1}, \ldots, Q_{n}\right)$ form a union of towers, then there are restrictions on the order in which the points must be blown up, and it is easily seen that the resulting surface $\widetilde{V}$ and the divisors $E_{i}$ and $e_{i}$ are independent of that order.

Proposition 2.2. Let $S=\left\{P_{1}, \ldots, P_{r}\right\}$ be a union of towers in $\mathbb{P}^{2}$ with the points numbered so that the index of any point is greater than the index of its source. Let $X$ be the surface obtained by sequentially blowing up the points of $S$, and let $E_{i}$ and $e_{i}$ respectively be the strict and total exceptional divisors on $X$. Let $D$ be a divisor on $X$.

(1) If $P_{i}$ is the source of some other point $P_{j}$, then $E_{i}=e_{i}-e_{j}$.

(2) Pic $X \cong \mathbb{Z}^{7}$, generated by $\ell, e_{1}, \ldots, e_{6}$. 
(3) The intersection pairing on $X$ is given by $\ell^{2}=1$, $\ell \cdot e_{i}=0, e_{i} \cdot e_{j}=-\delta_{i j}$.

(4) The canonical class of $X$ is $K=-3 \ell+\sum e_{i}$.

(5) The self-intersection of $D$ is $D^{2}=a^{2}-\sum b_{i}^{2}$.

(6) If $D$ is effective, its arithmetic genus is

$$
p_{a}(D)=\frac{1}{2}\left(D^{2}+D \cdot K\right)+1=\left(\begin{array}{c}
a-1 \\
2
\end{array}\right)-\sum\left(\begin{array}{c}
b_{i} \\
2
\end{array}\right) .
$$

Proposition 2.3. Let $S$ be a union of towers consisting of six points $P_{1}, \ldots, P_{6}$ of $\mathbb{P}^{2}$, no four collinear. Let $X$ be the surface obtained by blowing up $S$. Then any integral curve with negative self-intersection must be one of the following:

(1) an exceptional curve $E_{i}$, which has self-intersection -2 if there is a point of $S$ infinitely near to $P_{i}$ and -1 otherwise;

(2) the strict transform $F_{i j}$ of a line in $\mathbb{P}^{2}$ containing only the points $P_{i}$ and $P_{j}$ of $S$, which has self-intersection -1 ;

(3) the strict transform $F_{i j k}$ of a line in $\mathbb{P}^{2}$ containing the points $P_{i}, P_{j}$ and $P_{k}$ of $S$, which (if it exists) has self-intersection -2;

(4) the strict transform $G_{j}$ of a conic in $\mathbb{P}^{2}$ containing all of $S$ except $P_{j}$, which has self-intersection -1 ;

(5) the strict transform $G$ of a conic in $\mathbb{P}^{2}$ containing all of $S$, which (if it exists) has self-intersection -2 .

Proposition 2.4. Let $S$ be a union of towers consisting of six points $P_{1}, \ldots, P_{6}$ of $\mathbb{P}^{2}$, no four collinear. Let $X$ be the surface obtained by blowing up $S$. Then the anticanonical divisor $-K$ defines a morphism $\phi$, which is an isomorphism away from (-2)-curves and which collapses (-2)-curves, from $X$ to a normal rational cubic surface in $\mathbb{P}^{3}$ having only rational double points as singularities.

Conversely:

Theorem 2.5 [Nagata 1960, Theorem 8]. Let X be a normal cubic surface in $\mathbb{P}^{3}$ which is not a cone. Then the minimal desingularization $\tilde{X}$ of $X$ is isomorphic to $\mathbb{P}^{2}$ blown up at a set $S$ of 6 points which is a union of towers with no 4 points collinear. The morphism $\widetilde{X} \rightarrow X \subset \mathbb{P}^{3}$ is given by the linear system induced by the system of cubics through $S$.

Remark 2.5.1. A thorough treatment of Theorem 2.5 can be found in [O'Sullivan 1996]. Briefly, the idea of the proof is as follows: First, it follows easily that the singularities of $X$ are double points. Projecting from a singular point gives a birational map to $\mathbb{P}^{2}$ whose indeterminacy can be resolved by blowing up at the singular point. One then shows that the blown-up surface has only rational singularities, because these are the only singularities that can dominate a smooth 
point [Zariski and Samuel 1960]. Therefore, the original surface also has only rational singularities and thus can be desingularized by a sequence of blowings-up. The minimal desingularization $\tilde{X}$ dominates $\mathbb{P}^{2}$, and its canonical divisor has selfintersection 3 , since resolving rational double points does not affect the canonical divisor. Thus $\widetilde{X} \rightarrow \mathbb{P}^{2}$ must involve exactly six intermediate blowings-up. That $S$ is a union of towers with no four points collinear follows from the fact that the general hyperplane section of $X$ is a smooth elliptic curve.

\section{Relative constructions}

The main result of this section is that the process of blowing-up is compatible with flat families in the sense that, under conditions that are met for our applications, blowing up a flat family of schemes along a flat family of closed subschemes gives another flat family, and that taking a fibre of this family gives an identical result to taking a fibre and then blowing up. This will allow us to construct families of cubic surfaces from families of points in the plane and study limits of families of curves on these particular families of surfaces. A few algebraic preliminaries are first necessary.

The following lemma and its "sheafified" sequel are readily verified.

Lemma 3.1. Let $A \rightarrow B \rightarrow C$ be rings such that $B$ and $C$ are both flat over $A$. Let $M$ be an A-module. Then for all $i>0$,

$$
\operatorname{Tor}_{B}^{i}(B \otimes M, C)=0 .
$$

Proposition 3.2. Suppose $Z \stackrel{g}{\rightarrow} T$ and $Y \stackrel{f}{\rightarrow} T$ are flat morphisms and $Z \stackrel{h}{\rightarrow} Y$ is a morphism over $T$. Let $\mathscr{L}$ be a quasicoherent sheaf on $T$. Then, for all $i>0$,

$$
\operatorname{Tor}_{Y}^{i}\left(f^{*} \mathscr{L}, h_{*} \mathfrak{O}_{Z}\right)=0 .
$$

To proceed, we need a result from ring theory. Let $R$ be a ring. Recall that an ideal $I$ of $R$ is regular if it is generated by a regular sequence in $R ; I$ is perfect if $\operatorname{depth}(I)=\operatorname{pd}_{R}(R / I)$.

The significance of perfect ideals lies in the following result.

Theorem 3.3 [Balcerzyk and Józefiak 1989, III, Theorem 3.5.11]. Let $R$ be a Cohen-Macaulay ring, I an ideal of $R$ such that $\operatorname{pd}_{R}(R / I)$ is finite. Then $I$ is perfect if and only if $R / I$ is Cohen-Macaulay.

Theorem 3.4 (Auslander-Buchsbaum; see [Balcerzyk and Józefiak 1989, III, Theorem 3.5.6]). Let $R$ be a local ring with maximal ideal $\mathfrak{m}$, and let $M$ be a nonzero finitely-generated $R$-module of finite projective dimension. Then

$$
\operatorname{pd}_{R}(M)+\operatorname{depth}(\mathfrak{m} ; M)=\operatorname{depth}(\mathfrak{m}) .
$$


Corollary 3.5 [Balcerzyk and Józefiak 1989, III, Theorem 3.5.10]. Let $(R, \mathfrak{m})$ be a local ring, $I$ a regular ideal in $R$. Then for all $n \geq 1, I^{n}$ is a perfect ideal.

The following standard result will be used several times in the sequel.

Proposition 3.6 [Hartshorne 1977, III.9.7]. Let $X \stackrel{f}{\rightarrow} Y$ be a morphism of schemes, with $Y$ integral and regular of dimension 1. Then $f$ is flat if and only if every associated point $x \in X$ maps to the generic point of $Y$.

Corollary 3.7. Let $X$ be a Cohen-Macaulay scheme, $Z$ a closed subscheme which is a local complete intersection on $X$, that is, the ideal sheaf $\mathscr{I}=\Phi_{Z / X}$ is locally generated by $\operatorname{codim}(Z, X)$ elements.

(1) For each positive integers $n$, let $Z_{n}$ be the subscheme of $X$ defined by $\rrbracket^{n}$. Then $Z_{n}$ is a Cohen-Macaulay scheme.

(2) Suppose further that $Z$ is flat over a nonsingular curve $T$. Then for all $n, Z_{n}$ is flat over $T$.

Proof. (1) For any point $x \in X,\left(\Phi_{Z}\right)_{x}$ is a regular ideal in a Cohen-Macaulay local ring, so Corollary 3.5 can be used, which gives the result.

(2) By Proposition 3.6, flatness over $T$ is equivalent to having all associated points map to the generic point of $T$. Since $Z_{n}$ is Cohen-Macaulay from part (1), it has no embedded points, so the associated points are just the generic points of its components. But these are the components of $Z$, and so the fact that all of its generic points map to the generic point of $T$ forces the same to hold true for $Z_{n}$.

Proposition 3.8. Let $T$ be a smooth curve over $k$, and let $Y$ be an integral CohenMacaulay scheme which is flat and of finite type over $T$. Let $Z$ be a closed subscheme of $Y$ such that $Z$ is a local complete intersection on $Y$ and is also flat over $T$. Let $\tilde{Y}$ be the blowing-up of $Y$ along $Z$ with exceptional divisor $E$. Then $\tilde{Y}$ and $E$ are both flat over $T$, and for all $t \in T,(\tilde{Y})_{t}$ is the blowing-up of $Y_{t}$ at $Z_{t}$.

Proof. The blow-up map is birational, and $\widetilde{Y}$ is integral, so by Proposition 3.6, it is flat over $T$.

Now, let $Z_{n}$ be the subscheme of $Y$ defined by $\oiint_{Z}^{n}$ and tensor the exact sequence

$$
0 \rightarrow \mathscr{I}_{Z}^{n} \rightarrow \mathrm{O}_{Y} \rightarrow \mathrm{O}_{Z_{n}} \rightarrow 0
$$

by $\mathscr{O}_{Y_{t}}$. Since $Z_{n}$ is flat by Corollary 3.7, we can apply Proposition 3.2 to $\mathscr{L}=k(t)$ so that $\operatorname{Tor}_{Y}^{i}\left(\mathcal{O}_{Y_{t}}, \mathscr{O}_{Z_{n}}\right)=0$ for $i>0$. Hence we obtain the exact sequence

$$
0 \rightarrow \Phi_{Z}^{n} \otimes \mathrm{O}_{Y_{t}} \rightarrow \mathrm{O}_{Y_{t}} \rightarrow \mathrm{O}_{Z_{n}} \otimes \mathrm{O}_{Y_{t}} \rightarrow 0 .
$$

Let $\mathscr{F}=\mathscr{I}_{Z} \mathcal{O}_{Y_{t}}$. A priori, $\mathscr{g}^{n}$ is the image of $\mathscr{S}_{Z}^{n} \otimes \mathcal{O}_{Y_{t}}$ in $\mathscr{O}_{Y_{t}}$. But by the injectivity of the map on the left it follows that $\mathscr{I}_{Z}^{n} \otimes \mathcal{O}_{Y_{t}} \cong \mathscr{g}^{n}$. This shows that

$$
\operatorname{Proj} \oplus \mathscr{F}^{n} \cong \operatorname{Proj}\left(\mathscr{O}_{Y_{t}} \otimes \bigoplus \mathscr{I}_{Z}^{n}\right) \text {; }
$$


that is, blowing up is compatible with taking fibres. This is the last statement of the proposition.

Finally, since $E$ is of pure codimension 1 in $\widetilde{Y}$ and is of codimension 1 on every fibre of $\widetilde{Y}, E$ can have no components which lie over closed points of $T$. Thus, by Proposition 3.6, $E$ is flat over $T$.

\section{Families of blown-up surfaces}

We will now apply the results of the last section to a configuration of 6 points in $\mathbb{P}^{2}$, possibly infinitely near. For this section, we will be considering flat families of surfaces over a smooth curve, where a "smooth curve" is understood to be nonsingular, connected and of finite type over $k$ but not necessarily complete. The reason that it is important not to insist on completeness for our base schemes is that eventually (Proposition 4.11) it may become necessary to excise a finite number of closed points in order to make the results go through.

Proposition 4.1. Let $T$ be a (possibly nonprojective) smooth connected curve over $k$. Let $Z$ be a flat family of length-n, 0-dimensional schemes parametrized by $T$ such that, for all $t \in T$, the fibre $Z_{t}$ is reduced. Then there exists a surjective base extension $T^{\prime} \rightarrow T$ of curves such that $Z^{\prime}=Z \times_{T} T^{\prime}$ is the scheme-theoretic union of $n$ disjoint families of points parametrized by $T^{\prime}$.

Proof. Since each fibre of $Z$ is a union of reduced points, $Z$ is flat and unramified over $T$, so (by [Hartshorne 1977, III, Ex. 9.4]) $Z$ is smooth over $T$. Therefore $Z$ is the disjoint union of a number of smooth curves $Z_{i}$. Let $K$ be the function field of $T$, and let $K_{i}$ be the function field of $Z_{i}$.

Let $K_{1}^{\prime}$ be a splitting field for $K_{1}$ over $K$. Then tensoring the field extension $K \rightarrow K_{1}$ with $K_{1}^{\prime}$ gives $K_{1}^{\prime} \rightarrow\left(K_{1}^{\prime}\right)^{n_{1}}$; therefore, if $T_{1}^{\prime}$ is the normalization of $T$ in $K_{1}^{\prime}, Z_{1} \times_{T} T_{1}^{\prime} \cong \bigcup^{n_{1}} T_{1}^{\prime}$; that is, the base-extended family is the union of $n_{1}$ disjoint families of points. Now proceed in this manner to the other $Z_{i}$, finally producing a curve $T^{\prime}=T_{n}^{\prime}$ over which all of the $Z_{i}$ have been separated into disjoint families of points.

Example 4.1.1. Let $R=k[x]_{\left(x^{3}-x\right)}$ and $S=\left(R[y] / y^{2}-\left(x^{3}-x\right)\right)_{y}$, so the family of pairs of points given by the map $R \rightarrow S$ corresponds to the unramified part of a map of degree 2 from an elliptic curve to $\mathbb{P}^{1}$. (In this case, of course, the extension of function fields is already Galois and the curve normal.) Then tensoring by $S$ gives $S \rightarrow S[z] / z^{2}-y^{2} \cong S \oplus S$; we have thus base-extended the family to two disjoint families.

Proposition 4.2. Let $T$ be a smooth curve over $k$, let 0 be a (closed) point of $T$, and let $Y \rightarrow T$ be a flat family of smooth surfaces over $k$. Let $Z \subset Y$ be a family of length-n schemes such that $Z_{t}$ is a union of $n$ distinct points for $t \neq 0$. Then there 
exists a base extension $T^{\prime} \rightarrow T$ such that $Z^{\prime}=Z \times T^{\prime}$ is equal to the union of $n$ smooth irreducible components.

Proof. The base extension $T^{\prime} \rightarrow T \backslash\{0\}$ exists by Proposition 4.1. To make the base extension to all of $T$, consider the map of complete nonsingular curves induced by the base extension $T^{\prime} \rightarrow T \backslash\{0\}$ and augment $T^{\prime}$ by one of the preimages of 0 .

Definition 4.3. A 0-dimensional subscheme $Z$ of a surface $Y$ is curvillinear if $Z$ is contained locally in a smooth curve on $Y$.

Proposition 4.4. Let $T$ be a smooth curve over $k$ with special point 0 , and let $Y \rightarrow T$ be a flat family of smooth surfaces over $k$. Let $Z \subset Y$ be a family of length-n schemes such that $Z_{t}$ is reduced for $t \neq 0$ and such that the scheme $Z_{0}$ is curvillinear with base locus $S_{0}$. Then $S_{0}$ is a union of towers, and the fibre $Y_{0}$ is isomorphic to the sequential blowing-up of $Y$ at the points of the base locus $S_{0}$.

Proof. $S_{0}$ is a union of towers, since any other base locus (satisfying the proximity inequalities) has a coefficient $\geq 2$ for some point, which forces a curve containing the base locus to have a multiple point. If necessary, base-extend $T$ as in Proposition 4.2 so that $Z$ is the union of $n$ disjoint copies of $T$ away from 0 . Label the components of $Z$ as $P_{1}, P_{2}, \ldots, P_{n}$ and proceed by induction on $n$ :

For $n=1$, we can treat the point 0 just as any other point in Proposition 4.2. Notice that the blowing-up is a flat family of surfaces by Proposition 3.8.

Now let $Q=\left(P_{1}\right)_{0}$, the point at which $P_{1}$ meets $Y_{0}$. Consider the following exact diagram of $O_{Y}$-modules.

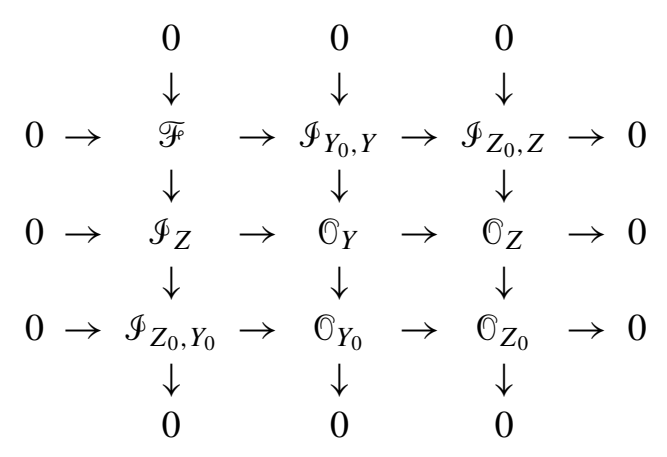

Choose $O(1)$ very ample on $Y$ and take $m \gg 0$ such that the general element of $H^{0} \mathscr{I}_{Z_{0}, Y_{0}}(m)$ is smooth and $H^{1} \mathscr{F}(m)=0$. Let $s \in H^{0} \Phi_{Z}(m)$ restrict to a smooth element of $H^{0} \Phi_{Z_{0}, Y_{0}}(m)$. Then $s$ is smooth at $Q$, since $Y_{0}$ is locally defined by a generator of the maximal ideal (coming from the uniformizing parameter at 0 ) in a regular local ring. Therefore $s$ defines a locally smooth surface $W$ in $Y$ such that $Z \subset W$. Blow up $Y$ at $P_{1}$ to obtain $Y^{1}$. On $Y^{1}$, the strict transform $\widetilde{W}$ of $W$ contains the strict transforms of $P_{2}, \ldots, P_{n}$. Further, the surface $\left(Y^{1}\right)_{0}$ is the blowing-up of $Y$ at $Q$ by Proposition 3.8, and the ideal of $\widetilde{W}_{0}$ is contained in the transform 
$\mathscr{I}_{Z}^{Y^{1}}$ of $\mathscr{I}_{Z}$, since each ideal differs from its total transform by exactly one copy of the exceptional divisor. $\Phi_{Z}^{Y^{1}}$ is a complete ideal corresponding to the base locus $S_{0} \backslash Q$ on $Y^{1}$. Now apply induction: $X \rightarrow Y^{1}$ has the property that $X_{0} \rightarrow\left(Y^{1}\right)_{0}$ is isomorphic to the sequential blowing-up of the points of $S_{0} \backslash\{Q\}$, and $\left(Y^{1}\right)_{0} \rightarrow Y_{0}$ is the sequential blowing-up of $Q$.

Remark 4.4.1. Note that the families $P_{i}$ can be blown up in any order at all, but the points $Q_{i}$ of $S_{0}$ have conditions imposed on the order in which they can be blown up, namely that "lower" points on any tower must be blown up before "higher" ones.

Remark 4.4.2. If $Q_{i}$ is in some tower with successors $Q_{i_{1}}, \ldots, Q_{i_{r}}$, then $e_{i}=$ $E_{i}+E_{i_{1}}+\cdots+E_{i_{r}}$, since at each successive blowing-up, the divisor will pick up exactly one copy of the new exceptional curve.

Proposition 4.5. Let $T$ be a nonsingular curve (as usual, not necessarily complete) over $k$ with point 0 , let $Y \rightarrow T$ be a flat family of smooth projective surfaces over $k$. Let $Z \subset Y$ be a flat family of curvillinear length-n subschemes of $Y_{t}$ such that $Z_{t}$ is a union of $n$ distinct points for $t \neq 0$. Suppose further that $Z=\bigcup_{\sigma \in \Sigma} P_{\sigma}$, where $\Sigma$ is a set of cardinality $n$ and each $P_{\sigma} \cong T$. Let $S_{0}$ be the base locus of $Z_{0}$, which consists of $n$ points with coefficient 1 by Proposition 4.4. Then any choice of numbering $P_{1}, \ldots, P_{n}$ of the $P_{\sigma}$ establishes a unique numbering $Q_{1}, \ldots, Q_{n}$ of the points of $S_{0}$ such that

(1) for all $i$ the fibre over 0 of the scheme $Y^{i}$ obtained by blowing up $P_{1}, \ldots, P_{i}$ sequentially is isomorphic to the sequential blowing-up of $Y_{0}$ at $Q_{1}, \ldots, Q_{i}$;

(2) if $Q_{i}=s\left(Q_{j}\right)$, then $i<j$;

(3) $Q_{i}$ is an ordinary point of $Y_{0}^{i-1}$ and $Q_{i}=P_{i} \cap Y_{0}^{i-1}$;

(4) on $X=Y^{n}$, the surface obtained by sequentially blowing up the $P_{i}$, the restriction of the strict transform of $P_{i}$ to $X_{0}$ is the total exceptional divisor $e_{i}$ corresponding to the point $Q_{i}$.

Proof. For each $i$ in sequence, take $Q_{i}$ to be the lowest (possibly infinitely near) point not yet blown up on the tower supported at the (closed) point where $P_{i}$ meets $Y_{0}$. This is clearly the only ordering that could possibly satisfy the desired conditions. By definition, the second is satisfied, and by Proposition 4.4, the first and third are satisfied.

For the last item, since neither of the divisors in question is going to be influenced by families of points that do not become infinitely near to $Q_{i}$, we may assume that $Q_{1}, \ldots, Q_{n}$ form a tower. Further, each divisor will consist only of divisors from subsequent points, so we may as well assume that $i=1$. If $n=1$, there is really nothing to prove, so assume that $n>1$. As in Proposition 4.4, on 
the blow-up $Y^{1}$ the families $P_{2}, P_{3}, \ldots, P_{n}$ all meet the exceptional divisor $E_{1}$ at the (ordinary) point $Q_{2}$ on $Y_{0}^{1}$. Therefore when we blow up $P_{2}$ to get the surface $Y^{2}$, the divisor $E_{1}$ gets the point $Q_{2}$ blown up, so that the strict transform of $E_{1}$ meets $E_{2}$ in the new exceptional line above 0 . The remaining $P_{i}$ all pass through a particular point on this line, so on blowing up $P_{3}$, we see that the strict transform of $E_{1}$ picks up yet another line above 0 . Similarly, each $P_{i}$ contributes a line, so that on the final surface $X=Y^{n}$ we will have $\left(e_{1}\right)_{0}=E_{1}+\cdots+E_{n}=e_{1}$ on the surface $X_{0}$.

Example 4.5.1. For an illustration, let $T=\mathbb{A}^{1}=\operatorname{Spec} k[t]$ and let $Z$ consist of two families of points in $\mathbb{A}^{2}$ given respectively by $(t, 0)$ and $(0, t)$. To find the ideal for $Z$, note that away from $t=0$ it is given by the product of the ideals of the families. This product is the ideal

$$
\begin{aligned}
(x-t, y)(y-t, x) & =\left(x y, y^{2}-t y, x^{2}-t x, x y-t x-t y+t^{2}\right) \\
& =\left(x y, y^{2}-t y, x^{2}-t x, t x-t y+t^{2}\right) \\
& =\left(x y, y^{2}-t y, x^{2}-t x, t(t-x-y)\right) .
\end{aligned}
$$

To find the ideal of the flat family, then, we need to throw into the ideal any elements killed by $t$, in this case $t-x-y$. Therefore the ideal for $Z_{0}$ is $I_{0}=\left(x^{2}, x y, y^{2}, x+\right.$ $y)=\left(x^{2}, x+y\right)$, and the associated base locus is $Q_{1}+Q_{2}$, where $Q_{1}$ is the origin and $Q_{2}$ is the tangent direction associated to the line $x+y=0$.

Now, blowing up the ideal $(x-t, y)$ gives (for the appropriate open affine) the ring map

$$
k[x, y, t] \stackrel{y \mapsto w(x-t)}{\longrightarrow} k[x, w, t] .
$$

The total transform of the family $(x, y-t)$ on this new surface is given locally by the ideal

$$
(w(x-t)-t, x)=(t(w+1), x)
$$

so that the strict transform is given by $(x, w+1)$. Therefore this second family meets the fibre over $t=0$ at the point on the exceptional curve $x=0$ where $w+1=0$, corresponding to the tangent direction $x+y=0$. Therefore, blowing up in the order we did established the correspondence

$$
(x-t, y) \leftrightarrow Q_{1},(x, y-t) \leftrightarrow Q_{2} .
$$

But by the symmetry of the variables, it is clear that blowing up the points in the other order would have reversed the correspondences.

Having blown up $(x-t, y)$, we now blow up the strict transform $(x, w+1)$ of the second family. This gives (again on the interesting open affine) the ring map

$$
k[x, w, t] \stackrel{x \mapsto r(w+1)}{\longrightarrow} k[r, w, t] .
$$


The composite blowing-up on the special fibre looked like

$$
k[x, y] \stackrel{y \mapsto w x}{\longrightarrow} k[x, w] \stackrel{x \mapsto r(w+1)}{\longrightarrow} k[r, w],
$$

which is the blowing-up of the origin followed by the blowing-up of the tangent direction $x+y$. Note that the surfaces over $t=0$ obtained by blowing up the two families of points in the other order are isomorphic, but that this isomorphism does not extend over any open neighborhood of 0 .

We can calculate the divisor class group of the threefold $X$ obtained from the blowing-up of a family of planes at a family of points by using the following, which is an adaptation of [Hartshorne 1997, Proposition 1.1].

Proposition 4.6. Let $T$ be an irreducible nonsingular curve over $k$ (again not necessarily complete). Let $X \rightarrow T$ be a projective flat family of surfaces over $T$ such that $H^{1}\left(O_{X_{t}}\right)=0$ and $X_{t}$ is smooth for all $t \in T$. If $1 \in T$ is any closed point, then there is an exact sequence

$$
0 \rightarrow \operatorname{Pic} T \stackrel{f_{*}}{\rightarrow} \operatorname{Pic} X \stackrel{\rho}{\rightarrow} \operatorname{Pic} X_{1},
$$

where $X_{1}$ denotes the fibre over 1 and $\rho$ is the restriction map.

Proof. By Stein factorization [Hartshorne 1977, III, Corollary 11.5], the map $f$ factors as the composition

$$
X \stackrel{f^{\prime}}{\rightarrow} Y=\operatorname{Spec} f_{*} O_{X} \stackrel{g}{\rightarrow} T,
$$

where $f^{\prime}$ has connected fibres and $g$ is finite. Since $f$ has connected fibres, this forces the fibres of $g$ to be connected as well, so that $g$ must be an isomorphism. Therefore $f_{*} \mathrm{O}_{X}=\mathrm{O}_{T}$. Then by the Projection Formula [Hartshorne 1977, II, Exercise 5.1], $f_{*} f^{*} \mathscr{L}=\mathscr{L}$ for any invertible sheaf $\mathscr{L}$ on $T$. This shows that the map $f_{*}$ on Pic is injective.

For exactness in the middle, suppose that $\mathscr{F}$ is an invertible sheaf on $X$ and that $\mathscr{F}_{1}=\mathscr{F}_{\mathbb{P}} \otimes \mathcal{O}_{X_{1}} \cong \mathcal{O}_{X_{1}}$. Since then $H^{1}\left(\mathscr{F}_{1}\right)=0$, the theorem on cohomology and base change [Hartshorne 1977, III, Theorem 12.11] shows that $R^{1} f_{*} \mathscr{F}=0$ in a neighborhood of $1, f_{*} \mathscr{F}$ is locally free in a neighborhood of 1 , and $\left(f_{*} \mathscr{F}\right) \otimes k(1) \cong$ $H^{0}\left(\mathscr{F}_{1}\right)=k$. Hence $f_{*} \mathscr{F}$ is invertible in a neighborhood of 1 .

Consider the natural map $f^{*} f_{*} \mathscr{F} \rightarrow \mathscr{F}$. This map restricts to an isomorphism on the fibre $X_{1}$, and it is therefore an isomorphism on a neighborhood of $X_{1}$. By properness, there is a neighborhood $V$ of 1 in $T$ such that $f^{*} f_{*} \mathscr{F} \cong \mathscr{F}$ on $f^{-1}(V)$, and hence $\mathscr{F}$ is in the image of Pic $V$ over this open set. 
Finally, let $P$ be any point of $T \backslash V$, and let $T^{\prime}=V \cup P, X^{\prime}=f^{-1} T^{\prime}$. By [Hartshorne 1977, II, 6.5], there is an exact sequence

$$
\mathbb{Z} \rightarrow \operatorname{Pic} X^{\prime} \rightarrow \operatorname{Pic} f^{-1} V \rightarrow 0
$$

where the first map sends 1 to the class of $X_{P}$ in Pic $X^{\prime}$. Since $X_{P}=f^{*}(P)$, and the kernel of Pic $X^{\prime} \rightarrow$ Pic $X_{1}$ differs from the kernel of Pic $f^{-1}(V) \rightarrow$ Pic $X_{1}$ by a multiple of $X_{P}$, this shows that any $\mathscr{F}$ on $X^{\prime}$ which maps to 0 in Pic $X_{1}$ comes from $f^{*}$. Proceeding in this manner on the other points of $T \backslash V$ gives the result.

Proposition 4.7. Let $Z$ be a flat family of length-n schemes in $\mathbb{P}_{T}^{2}$ such that $Z_{t}$ is the union of $n$ distinct points for $t \neq 0$. By Proposition 4.2, we can replace $T$ by a base extension so that $Z$ is the union of $n$ smooth irreducible components. Number these components $P_{1}, \ldots, P_{n}$ and let $X$ the resulting family of blown-up surfaces. By Proposition 4.5, the points of the base locus associated to $Z_{0}$ have an ordering $Q_{1}, \ldots, Q_{n}$ imposed on them by the numbering of the $P_{i}$.

(1) Pic $X / \operatorname{Pic} T$ is a free abelian group with generators $\ell_{X},\left(e_{1}\right)_{X}, \ldots,\left(e_{n}\right)_{X}$, where $\ell_{X}=\pi^{*} \mathbb{O}_{\mathbb{P}_{T}^{2}}(1)$ and $\left(e_{i}\right)_{X}$ is the strict transform of $P_{i}$.

(2) Denote by $\ell_{t}$ and $\left(e_{i}\right)_{t}$, respectively, the total transforms of $\mathrm{O}_{\mathbb{P}_{k(t)}^{2}}$ (1) and the strict exceptional curves $e_{i}$ on the surface $X_{t}$. Then for each $t \in T$, the natural restriction map Pic $X \rightarrow \operatorname{Pic} X_{t}$ factors to give an isomorphism Pic $X /$ Pic $T \rightarrow$ Pic $X_{t}$ taking $\ell_{X}$ to $\ell_{t}$ and $\left(e_{i}\right)_{X}$ to $\left(e_{i}\right)_{t}$.

Proof. First, the divisor $\left(e_{i}\right)_{X}$ restricts to $\left(e_{i}\right)_{t}$ on each $X_{t}$ by Proposition 4.5; clearly $\ell_{X}$ restricts to $\ell_{t}$ as well. Proposition 4.6 shows that the restriction map gives an injection Pic $X / \operatorname{Pic} T \rightarrow \operatorname{Pic} X_{t}$ for each fibre, but these maps are actually surjective, since $\ell$ and the $e_{i}$ generate the Picard groups for the blown-up surfaces.

Remark 4.7.1. As with a single blown-up surface, we will write a divisor class $a \ell-b_{1} e_{1}-\cdots-b_{n} e_{n}$ on $X$ as the $n$-tuple $\left(a ; b_{1}, \ldots, b_{n}\right)$.

Definition 4.8. I will refer to a length- 6 subscheme of $\mathbb{P}^{2}$ as general if it is reduced, does not lie on a conic, and meets no line in length $\geq 3$. Such a subscheme is almost general if it is curvillinear and meets no line in length $\geq 4$.

Remark 4.8.1. By [Hartshorne 1977, V, Section 4], we see that general subschemes are those whose base loci give smooth cubics when blown up.

Theorem 4.9 [Fogarty 1968, Theorem 2.4]. Let $d$ be a positive integer. Then the family of 0-dimensional subschemes of $\mathbb{P}^{2}$ of length d is irreducible.

Corollary 4.10. Let $S$ be any 6-point base locus on $\mathbb{P}^{2}$ which is a union of towers such that no four points of $S$ are contained in a line, and let $Z$ be the associated scheme. Then there exists a smooth curve $T$ with point 0 and a flat family of schemes $Z_{T}$ over $T$ such that $Z_{t}$ is general for $t \neq 0$ and $Z=Z_{0}$. 
Proposition 4.11. Let $T$ be a smooth curve, as usual not necessarily complete. Let

$$
Z=P_{1} \cup \cdots \cup P_{6} \subset \mathbb{P}_{T}^{2}, \quad P_{i} \cong T,
$$

be a family over $T$ of length- 6 subschemes of $\mathbb{P}^{2}$ such that $Z_{t}$ is general for $t \neq 0$ and $Z_{0}$ is almost general. Let $\widetilde{X}$ be the scheme obtained by blowing up $\mathbb{P}_{T}^{2}$ sequentially at the curves $P_{1}, \ldots, P_{6}$. Then (possibly after shrinking $T$ to a neighborhood of 0 ) the morphism

$$
\widetilde{X} \stackrel{\phi}{\rightarrow} \mathbb{P}_{T}^{3}
$$

given by the complete linear system $(3 ; 1,1,1,1,1,1)$ has image $Y$ which is a flat family of normal cubic surfaces in $\mathbb{P}^{3}$ such that for each $t, Y_{t}$ is isomorphic to the image in $\mathbb{P}^{3}$ of the surface obtained by sequentially blowing up the base locus associated to $Z_{t}$ (which is just a reduced set of points for $t \neq 0$ ).

Proof. Let $\mathscr{L}$ be the invertible sheaf on $\widetilde{X}$ corresponding to the divisor class (3; $1,1,1,1,1,1)$. First, I claim that $H^{1}\left(\mathscr{L}_{0}\right)=0$. To see this, let $H$ be a smooth effective divisor in $\left|\mathscr{L}_{0}\right|$ on $\widetilde{X}_{0}$ (these exist since the image of $\widetilde{X}_{0}$ under $\mathscr{L}_{0}$ has only isolated singularities) and consider the exact sequence of sheaves on $\widetilde{X}_{0}$

$$
0 \rightarrow \mathrm{O}_{\widetilde{X}_{0}} \rightarrow \mathscr{L}_{0} \rightarrow \mathrm{O}_{H}\left(\mathscr{L}_{0}\right) \rightarrow 0 .
$$

Now, $H^{1} \widetilde{O}_{\widetilde{X}}=0$, and $H^{1} \widehat{O}_{H}\left(\mathscr{L}_{0}\right)=0$ because $\left.\mathscr{L}_{0}\right|_{H}$ is a divisor of degree 3 on an elliptic curve. This forces $H^{1}\left(\mathscr{L}_{0}\right)=0$.

Now, again by cohomology and base change, $f_{*} \mathscr{L}$ is free (of rank 4 ) in some neighborhood of 0 ; therefore, shrinking $T$ if necessary, we may assume that there are global sections of $\mathscr{L}$ which restrict to a generating set of $\mathscr{L}_{t}$ on $\widetilde{X}_{t}$ for each $t \in T$. Then $\mathscr{L}$ is generated by global sections, since at any stalk a nongenerator restricts to a nongenerator on its fibre.

Thus $\mathscr{L}$ gives a morphism $\phi: \widetilde{X} \rightarrow \mathbb{P}_{T}^{3}$. Since the restriction $\mathscr{L}_{t}$ of $\mathscr{L}$ is the linear system $(3 ; 1,1,1,1,1,1)$ on $\widetilde{X}_{t}$, it gives the map from $\widetilde{X}_{t}$ into $\mathbb{P}^{3}$ with image the normal cubic surface which comes from the blowing-up of $Z_{t}$.

Remark 4.11.1. It is via this morphism $\phi$ that we can extract information about divisors on the normal cubic surface $X_{0}$ from information about the family $\tilde{X}$ and Proposition 4.7. Roughly speaking, one would like to take a divisor $C_{0}$ on $X_{0}$, translate it into a divisor $\widetilde{C}_{0}$ on $\widetilde{X}_{0}$, identify the divisor type, and use this information to express $C_{0}$ as the limit of a flat family of curves on smooth cubic surfaces. The difficulty with this method is that the actual limit of the flat family so obtained may have embedded points, so the challenge is to guarantee somehow that one can find a divisor class on $\widetilde{X}_{0}$ giving $C_{0}$ such that the limit of the resulting family is without embedded points. 
Example 4.11.2. Consider the family $\tilde{X}$ of surfaces obtained from the blowing-up of $\mathbb{P}_{T}^{2}$ at the family $Z=P_{1} \cup \cdots \cup P_{6}$, where the $P_{i}$ are ordinary and general except that $\left(P_{1}\right)$ and $\left(P_{2}\right)$ meet in $\mathbb{P}_{k(0)}^{2}$, and $\left(P_{3}\right)$ and $\left(P_{4}\right)$ meet at another point of $\mathbb{P}_{k(0)}^{2}$, so that the base locus $Q_{1}, \ldots, Q_{6}$ associated to $Z_{0}$ (in the sense of Proposition 4.5) has $Q_{2}$ infinitely near to $Q_{1}$ and $Q_{4}$ infinitely near to $Q_{3}$. Upon sequentially blowing up, we find that $\widetilde{X}_{0}$ has two $(-2)$-curves which do not meet, so that in the family $X \subset \mathbb{P}_{T}^{3}, X_{t}$ is smooth for $t \neq 0$ and $X_{0}$ has two isolated singular points. (In fact, each is a rational double point of type $\mathbf{A}_{1}$, analytically isomorphic to the "cone singularity" $x y-z^{2}$.) Consider the line $F_{13} \subset \mathbb{P}_{k(0)}^{2}$ containing $Q_{1}$ and $Q_{3}$. Its strict transform in $\widetilde{X}_{0}$ will be mapped under the restriction of $\phi$ to a line $L_{0}$ in $\mathbb{P}^{3}$, since it is a limit of the strict transforms of the lines $(1 ; 1,0,1,0,0,0)$ on the smooth cubic surfaces $X_{t}$. Notice that $L_{0}$ contains both of the singular points of $X_{0}$.

Now consider the multiplicity- 2 structure $2 L_{0}$ on $L_{0} \subset X_{0}$, defined by throwing out embedded points from the subscheme of $X_{0}$ defined by $\Phi_{L_{0}, X_{0}}^{2}$. I claim that $2 L_{0}$ is actually contained in a plane. To see this, choose any point of $L_{0}$ besides the two singularities of $X_{0}$ and consider the plane section of $X_{0}$ containing $L_{0}$ and the normal direction at the chosen point. This plane section has 3 singular points lying on $L_{0}$, and it is easily shown that any plane curve of degree 3 having 3 singular points along a line must contain the 2-structure on that line, so our chosen plane section contains $2 L_{0}$.

Note that each of the families $F_{13}, F_{14}, F_{23}, F_{24}$ on the smooth cubics specialize to $L_{0}$, where $F_{i j}$ is the family of lines on the smooth cubics coming from lines through $P_{i}$ and $P_{j}$ in the plane. Therefore, there are a number of ways to try to write $2 L_{0}$ as a (flat) limit, and all of them will agree with $2 L_{0}$ generically. However, for example, the limit of $F_{13}+F_{14}$ has an embedded point, since the fibres are disjoint for $t \neq 0$, making the arithmetic genus -1 , so $2 L_{0}$ is not the flat limit of this family. On the other hand, $F_{13}$ and $F_{24}$ meet in every fibre, so the arithmetic genus of $F_{13}+F_{24}$ is 0 , which is the arithmetic genus of $2 L_{0}$. By [Hartshorne 1977, Theorem III.9.7], $L_{0}$ is the flat limit of the family $F_{13}+F_{24}$.

Proposition 4.12. Let $Y$ be a family of smooth surfaces over a nonsingular curve $T$ with point $0 \in T$. Let $\mathscr{L}$ be a divisor class on $Y$ whose restriction $\mathscr{L}_{0}$ to $Y_{0}$ is represented by an effective divisor $D_{0}$ such that either

(1) $H^{1}\left(Y_{0}, \mathscr{L}_{0}\right)=0$ or

(2) $H^{2}\left(Y_{0}, \mathscr{L}_{0}\right)=0$ and $H^{1}\left(Y_{t}, \mathscr{L}_{t}\right)$ is constant in a neighborhood of 0 .

Then, possibly after shrinking $T$ to an open neighborhood of 0 , the divisor class $\mathscr{L}$ is represented by an effective divisor $D$ on $Y$ without vertical components whose restriction to $Y_{0}$ is equal to $D_{0}$. 
The following is needed for the vanishing of certain cohomology groups.

Lemma 4.13. Let $X$ be a smooth surface, and let $C \subset X$ be a reduced Cartier divisor such that each irreducible component of $C$ has negative intersection with the canonical divisor $K_{\widetilde{\mathbb{P}}}$ of $\widetilde{\mathbb{P}}$. Then $H^{1} \widehat{O}_{C}(C)=0$.

Proof. Let $\omega_{C}$ be the dualizing sheaf of $C$, and let $\omega_{X}$ be the canonical sheaf of $X$. Then $\left.\omega_{C} \cong \omega_{X}(C)\right|_{C}$. By Serre duality, $H^{1} \widehat{C}_{C}(C) \perp H^{0} \omega_{C}(-C)=H^{0}\left(\left.\omega_{X}\right|_{C}\right)$. Therefore it suffices to show that $H^{0}\left(\left.\omega_{X}\right|_{C}\right)=0$.

First, assume that $C$ is integral. Then $\left.\omega_{X}\right|_{C}$ is a Cartier divisor of negative degree on the integral curve $C$, so $H^{0}\left(\left.\omega_{X}\right|_{C}\right)=0$.

Now suppose that $C=\bigcup_{1}^{r} C_{i}$, where the $C_{i}$ are integral. Let $Y$ be the disjoint union of the $C_{i}$. Then the natural projection $Y \stackrel{f}{\rightarrow} C$ exhibits $\left.\omega_{X}\right|_{C}$ as a subsheaf of $f_{*}\left(\left.\bigoplus \omega_{X}\right|_{C_{i}}\right)$. Since $H^{0}\left(\left.\bigoplus \omega_{X}\right|_{C_{i}}\right) \cong H^{0}\left(f_{*}\left(\left.\bigoplus \omega_{X}\right|_{C_{i}}\right)\right)$ (see [Hartshorne 1977, III, Ex. 8.2], for example), and since $H^{0}\left(\left.\omega_{X}\right|_{C_{i}}\right)=0$ for all $i$ by the previous paragraph, $H^{0}\left(\left.\omega_{X}\right|_{C}\right)=0$ and the lemma is proved.

\section{The Main Theorem}

Configuration of (-2)-curves on the blown-up surface. In this section, I will identify the possible configurations of $(-2)$-curves on a surface obtained by blowing up a 6-point base locus in $\mathbb{P}^{2}$ which is a union of towers with no four base points collinear.

Definition 5.1. I will refer to a connected set of curves as a cluster. If a surface singularity arises from the collapsing of a cluster of $(-2)$-curves to a point, this singularity is sometimes labelled according to the Dynkin diagram of the configuration of the (-2)-curves; see Definition 5.1. One can show that the configuration of such (-2)-curves is an invariant of the analytic isomorphism class of the singularity (see [Lipman 1969] or [O'Sullivan 1996] for details). It is well-known that the intersection matrix of the set of $(-2)$-curves collapsing to a point is negativedefinite (see [Artin 1962]).

It is known [Artin 1966] that any rational double point surface singularity contained in $\mathbb{P}^{3}$ is of type $\mathbf{A}_{r}, \mathbf{D}_{r}, \mathbf{E}_{6}, \mathbf{E}_{7}$, or $\mathbf{E}_{8}$; for rational normal cubic surfaces, this list is shorter, namely $\mathbf{A}_{1}, \ldots, \mathbf{A}_{5}, \mathbf{D}_{4}, \mathbf{D}_{5}$, or $\mathbf{E}_{6}$ (see [Bruce and Wall 1979]).

Recall from [Artin 1966] that the fundamental cycle $\xi_{0}$ associated to a rational surface singularity is defined (locally) as the smallest exceptionally supported positive cycle on the minimal desingularization with the property that its intersection with each exceptional curve is nonpositive. Fundamental cycles for the rational double points above are calculated as follows. Note that I express each in terms of its coefficients in the free monoid on the exceptional curves $E_{i}$; thus, for example, 


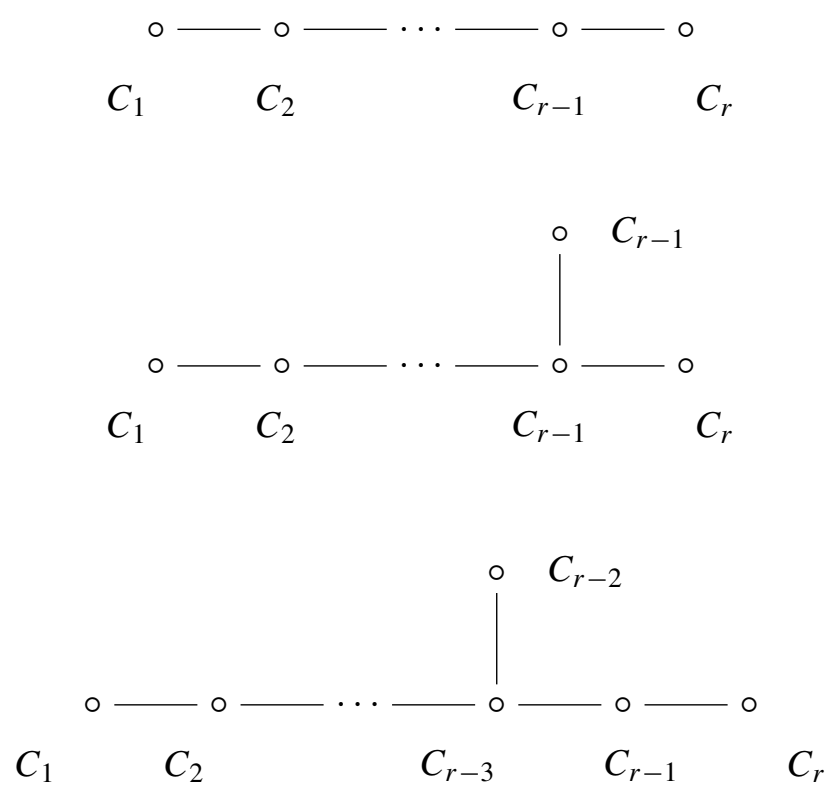

Figure 1. Top to bottom: Dynkin diagrams for $\mathbf{A}_{r}, \mathbf{D}_{r}$, and $\mathbf{E}_{r}$ configurations. The circles in the diagrams stand for curves, and a connecting line segment between two circles indicates that the corresponding curves intersect transversally at a single point.

$(2,3,1)$ stands for the divisor $2 E_{1}+3 E_{2}+E_{3}$. The curves are numbered as in Figure 1 .

- For $\mathbf{A}_{r}, \xi_{0}=(1,1, \ldots, 1)$.

- For $\mathbf{D}_{r}, \xi_{0}=(1,2,2,2, \ldots, 2,1,1)$.

- For $\mathbf{E}_{6}, \xi_{0}=(1,2,3,2,2,1)$.

- For $\mathbf{E}_{7}, \xi_{0}=(1,2,3,4,2,3,2)$.

- For $\mathbf{E}_{8}, \xi_{0}=(2,3,4,5,6,3,4,2)$.

Definition 5.2. Suppose that $\widetilde{\mathbb{P}}_{S}$ is a surface arising as the minimal resolution of a rational normal cubic surface in $\mathbb{P}^{3}$, and let $D$ be an effective divisor on $\widetilde{\mathbb{P}}_{S}$. $D$ has small intersection with fundamental cycles if, for each connected cluster $\left\{C_{1}, \ldots, C_{r}\right\}$ of $(-2)$-curves on $\widetilde{\mathbb{P}}_{S}$ with fundamental cycle $\xi_{0}, D$ has nonnegative intersection multiplicity with each of the $E_{i}$ and $D . \xi_{0} \leq 1$. (Equivalently, $D$ has nonzero intersection multiplicity with at most one of the curves in the cluster, and furthermore, if there is such a curve $C_{k}$, then $D . E_{k}=1$ and $C_{k}$ has coefficient 1 in $\xi_{0}$.) 
Proposition 5.3. Let $(R, \mathfrak{m})$ be a local ring defining a rational double point on a surface, let $\operatorname{Spec} S \stackrel{\pi}{\rightarrow} \operatorname{Spec} R$ be the minimal desingularization, with fundamental cycle $\xi_{0}$, and let $D \subseteq \operatorname{Spec} S$ be a nonexceptional smooth curve. Then the multiplicity of the scheme-theoretic image $\pi(D)$ at $\mathfrak{m}$ is equal to $D . \xi_{0}$.

Proof. Artin [1966, proof of Theorem 4] shows that $\mathscr{I}\left(\xi_{0}\right)=\mathfrak{m} \cdot S$. Now, restricting the map $R \rightarrow S$ to $D$ gives a map from the local ring $(A, \mathfrak{q})$ of the scheme-theoretic image of $D$ on Spec $R$ to a semilocal ring $B$ whose maximal ideals $\mathfrak{p}_{i}$ correspond to the points of $D$ on the exceptional divisor. Now, each localization $B_{\mathfrak{p}_{i}}$ is a discrete valuation ring, and the multiplicity $e_{i}$ of $\mathfrak{p}_{i}$ in the primary decomposition of $\mathfrak{m} B$ is precisely the coefficient of the component of the exceptional curve containing the closed point $p_{i}$ in $\xi_{0}$, from Artin's above observation. Now, apply Corollary 1 to [Zariski and Samuel 1960, vol. II, VII, Theorem 24] to conclude that

$$
m_{\mathfrak{m}}(\pi(D))=\sum m_{p_{i}}(D) \cdot e_{i}
$$

From the preceding considerations, this last sum is equal to $D . \xi_{0}$.

Corollary 5.4. Let $\widetilde{\mathbb{P}}_{S} \stackrel{\pi}{\rightarrow} S$ be the minimal resolution of a rational normal cubic surface in $\mathbb{P}^{3}$, and let $D$ be an effective divisor on $\widetilde{\mathbb{P}}_{S}$ having small intersection with fundamental cycles. Then $D \rightarrow \pi(D)$ is an isomorphism.

Proof. Locally, the map on $D$ is a birational morphism of smooth curves.

Proposition 5.5. Let $S$ be a 6-point base locus in $\mathbb{P}^{2}$ which is a union of towers such that no four points of $S$ are collinear. Let $\widetilde{\mathbb{P}}_{S}$ be the surface obtained by blowing up $S$ sequentially. Let $D$ be an effective divisor on $\widetilde{\mathbb{P}}_{S}$ having nonnegative intersection with each (-2)-curve. Then there exists an effective divisor $E$, supported on (-2)curves, such that $D+E$ has small intersection with fundamental cycles.

Proof. Clearly, it suffices to consider each connected cluster of (-2)-curves separately. Let $\left\{C_{1}, \ldots, C_{r}\right\}$ be such a cluster, and let $a_{i}=D . C_{i}$. When convenient, I will adopt the notation using ordered $r$-tuples for exceptionally supported divisors introduced above. I will also use square brackets to indicate intersection multiplicities with the -2-curves; thus, I would write that $D$ has intersection multiplicities $\left[a_{1}, \ldots, a_{r}\right]$.

First, suppose that the $C_{i}$ form an $\mathbf{A}_{r}, r \geq 1$. Proceed by induction on $\mathrm{s}=\Sigma a_{i}$. If $s \leq 1$, then $D$ has small intersection with the fundamental cycle, and we are done.

If $s \geq 2$, first suppose that there are two indices $i<j$ such that $a_{i}$ and $a_{j}$ are positive. Then add the divisor $C_{i}+C_{i+1}+\cdots+C_{j}$. This addition reduces $a_{i}$ and $a_{j}$ by 1 each and leaves the other intersection numbers unchanged; by induction, we are finished. 
Finally, if there is only one index $i$ such that $a_{i}$ is positive, then $a_{i} \geq 2$. Add a single copy of $E_{i}$ to $D$. Then there are two possibilities: If $1 \neq i \neq r$, then there are at least two entries, namely the $(i-1)^{s t}$ and the $(i+1)^{s t}$, in the new divisor which are nonzero, and we may proceed as in the last paragraph; if $i=1$ or $i=r$, then the sum of the entries has dropped, since the $i$-th has dropped by 2 and there is only one which has increased by 1 , and we are finished by induction.

For a $\mathbf{D}_{4}$, note that $\xi_{0} \cdot C_{i}=-\delta_{i 2}$. By adding copies of $C_{1}, C_{3}$, and $C_{4}$ to $D$, we may assume that each of $a_{1}, a_{3}, a_{4}$ is less than or equal to 1 ; and by adding a multiple of $\xi_{0}$, we may assume that $a_{2}=0$. By symmetry, it suffices to assume that $a_{1} \geq a_{3} \geq a_{4}$.

If $a_{3}=0$, then $D$ has small intersection with the fundamental cycle.

If $a_{1}=a_{3}=1, a_{4}=0$, then add the divisor $(1,1,1,0)$ to get a divisor with intersections $[0,0,0,1]$, which has small intersection with the fundamental cycle.

If $a_{1}=a_{3}=a_{4}=1$, then adding the divisor $(2,3,2,2)$ gives intersections $[0,0,0,0]$.

Thus the proposition is true for a $D_{4}$.

For $r>4$, observe that the curves $C_{2}, \ldots, C_{n}$ form a $D_{r-1}$, so by induction we may assume that there is an divisor $L$, supported on $C_{2} \cup C_{3} \cup \cdots \cup C_{n}$, such that $D^{\prime}=D+L$ has intersection number 1 with at most one of $C_{2}, C_{r-1}$, and $C_{r}$ and intersection number 0 with all other $C_{i}$ except $C_{1}$. Write the new intersection sequence as $\left[b_{1}, \ldots, b_{r}\right]$, and note that $b_{1} \geq a_{1} \geq 0$. If $b_{1} \geq 2$, we can reduce $b_{1}$ by 2 and leave the other $b_{i}$ unchanged by adding the divisor $(2,2, \ldots, 2,1,1)$, so we may assume that $b_{1}$ is either 0 or 1 .

If $b_{1}=b_{2}=0$, then we are done, as $D^{\prime}$ has small intersection with the fundamental cycle. If $b_{1}=1$ and all other $b_{i}$ are 0 , we are also done. If $b_{1}=$ $b_{2}=1$, then $D^{\prime}+\xi_{0}$ has small intersection with $\xi_{0}$. If $b_{1}=b_{r-1}=1$, then $D^{\prime}+C_{1}+C_{2}+\cdots+C_{r-1}$ has small intersection with $\xi_{0}$. Treat the case $b_{1}=b_{r}=1$ similarly.

This exhausts all possibilities, so the $\mathbf{D}_{r}$ case is complete.

In the case of an $\mathbf{E}_{6}$, notice that adding the fundamental cycle $\xi_{0}$ reduces the intersection number with $C_{4}$ by 1 and leaves the others unchanged; therefore, we can always assume that the intersection number of our divisor of interest with $C_{4}$ is 0 . From the $A_{r}$ case above, add an effective exceptionally supported divisor to $D$ in order to obtain a divisor $D^{\prime}$ whose intersection numbers with $C_{1}, C_{2}, C_{3}, C_{5}$, and $C_{6}$ are all 0 except for possibly one of them; if there are none, clearly we are done, and if $D^{\prime} . C_{1}=1$ or $D^{\prime} . C_{6}=1$, we are done as well, since these curves have multiplicity 1 in $\xi_{0}$. If $D^{\prime} . C_{2}=1$, add $(0,1,1,0,1,1)$; similarly for $C_{5}$. If D. $C_{3}=1$, add $(1,3,4,2,2,0)$. 
For an $\mathbf{E}_{7}$, note that adding the fundamental cycle reduces the intersection number with $C_{7}$ by 1 and leaves the others unchanged; as above, then, we need not worry about this intersection number. Also, note that $C_{1}, \ldots, C_{6}$ form a $\mathbf{D}_{6}$; therefore, we can find an effective divisor supported on these curves to add to $D$ in order to obtain $D^{\prime}$, whose sequence of intersection multiplicities is either: all 0 's, in which case we are done; $[1,0,0,0,0,0,0]$, which already has small intersection with the fundamental cycle; $[0,0,0,0,0,1,0]$, in which case we add $(2,4,6,8,4,6,3)$ to obtain all 0 's; or $[0,0, \ldots, 1,0,0]$, in which case we add $(0,1,2,3,2,3,2,1)$ to get back to $[1,0,0,0,0,0,0]$.

Finally, in the case of an $\mathbf{E}_{8}$, adding $\xi_{0}$ reduces the intersection number with $C_{1}$ and leaves the others unchanged. $C_{2}, \ldots, C_{8}$ form an $\mathbf{E}_{7}$, so from the previous case we may assume that we can add an appropriate divisor to $D$ and obtain $D^{\prime}$ whose sequence of intersection multiplicities is either all 0's (done) or [0, 1, 0, 0, 0, 0, 0, 0]. In this latter case, adding $(3,6,8,10,12,6,8,4)$ reduces the intersection multiplicities to all 0's. This finishes the proof.

Definition 5.6. Label the standard basis elements of $\mathbb{Z}^{r}$ as $\ell, e_{1}, \ldots, e_{r-1}$, and denote by $f_{i j}$ the elements $\ell+e_{i}+e_{j}$ for $i \neq j$. Define the Cremona transformation $\tau_{i j k}$ for $i, j, k$ all different to be the change of basis $\ell^{\prime}, e_{1}^{\prime}, \ldots, e_{n}^{\prime}$ where $\ell^{\prime}=l, e_{i}^{\prime}=$ $f_{j k}, e_{j}^{\prime}=f_{i k}, e_{k}^{\prime}=f_{i j}$, and $e_{n}^{\prime}=e_{n}$ for $n$ different from $i, j$, and $k$.

Remark 5.6.1. The reason I call this a Cremona transformation is that it is a generalization of the change of basis induced by the plane Cremona on the surface obtained by blowing up three noncollinear points, which makes sense whether the points are ordinary or infinitely near.

Lemma 5.7. Let $D$ be an element of $\mathbb{Z}^{7}$. Then, by a finite number of applications of the Cremona transformation $\tau_{123}$ and reorderings, one can produce a basis $\ell, e_{1}, \ldots, e_{6}$ for $\mathbb{Z}^{7}$ such that, with respect to this basis, the representation $D=a \ell-\sum b_{i} e_{i}$ satisfies

(1) $b_{1} \geq b_{2} \geq b_{3} \geq b_{4} \geq b_{5} \geq b_{6}$

(2) $a-b_{1}-b_{2}-b_{3} \geq 0$.

Proof. Label the standard basis elements for $\mathbb{Z}^{7}$ as $\ell, e_{1}, e_{2}, \ldots, e_{6}$. In order to avoid cumbersome notation, I will abuse notation and use the same names for elements of each basis produced on the way to the desired one. Define a bilinear form on $\mathbb{Z}^{7}$ as on a smooth cubic surface, $i$ @ $e . \ell^{2}=1, \ell . e_{i}=0, e_{i} . e_{j}=-\delta_{i j}$, and label the elements $f_{i j}=l-e_{i}-e_{j}, g_{j}=2 l-\sum_{i \neq j} e_{i}$. Then condition (2) is interpreted as $D . f_{12} \geq D . e_{3}$. In order for the $b_{i}$ to be in descending order, this forces $D . f_{i j}=a-b_{i}-b_{j} \geq D \cdot e_{3}=b_{3}$. Note also that D. $e_{1} \geq D \cdot e_{2} \geq D \cdot e_{3}$. Finally, $a-b_{i}-b_{j}-b_{k} \geq 0$ for any $i, j, k$, so $D . g_{m}=2 a-\sum_{i \neq m} b_{i} \geq b_{3}$ for all $g_{m}$. Therefore, with respect to our desired basis, the elements $e_{6}, e_{5}, e_{4}, e_{3}$ must have, 
in order, the four smallest intersections with $D$ among the set $\mathscr{Y}=\left\{e_{i}, f_{i j}, g_{k}\right\}$. I will show that an algorithm exists which accomplishes this.

By repeated applications of the Cremona transformation $\tau_{123}$ and reorderings, whatever element of $\mathscr{Y}$ having minimal intersection with $D$ can be brought to $e_{6}$. Then the remaining $e_{i}$ can be renumbered so that the $b_{i}$ are in descending order. Then, as in the previous paragraph, the element of $\mathscr{Y}$ which has the next minimal intersection with $D$ is $e_{5}, f_{12}$, or $g_{6}$.

If it is $e_{5}$, proceed to the next step in the algorithm.

If it is $f_{12}$, applying $\tau_{123}$ leaves $e_{4}, e_{5}$, and $e_{6}$ alone but takes $f_{12}$ to $e_{3}$. By renumbering, then, $e_{5}$ can be made to have next smallest intersection with $D$.

Finally, if it is $g_{6}$, apply $\tau_{123}$ so that $g_{6}$ becomes $f_{45}$; by renumbering, this will then become $f_{12}$, and we are in the previous case.

So far we have the desired $e_{6}$ and $e_{5}$. Again renumber the remaining $e_{i}$ so that the $b_{i}$ are descending. At this point, a priori again the candidates for the next smallest intersection with $D$ are $e_{4}, f_{12}$, and $g_{6}$, but I claim that we need not consider $g_{6}$. To see this, note that by choice of $e_{5}, D . e_{5} \leq D . f_{12}$, or in other words $b_{5} \leq a-b_{1}-b_{2}$. Substituting this inequality gives

$$
\begin{aligned}
D . g_{6} & =2 a-b_{1}-b_{2}-b_{3}-b_{4}-b_{5} \\
& \geq 2 a-b_{1}-b_{2}-b_{3}-b_{4}-\left(a-b_{1}-b_{2}\right) \\
& =a-b_{3}-b_{4} \\
& \geq a-b_{1}-b_{2} \\
& =D . f_{12} .
\end{aligned}
$$

Therefore, the next element of our basis is either $e_{4}$, in which case we can move on to the next step, or $f_{12}$, which is treated just like the second case in the step above and does not affect our $e_{5}$ or $e_{6}$.

The final step in the algorithm goes just like the previous one, since the Cremona transformation $\tau_{123}$ does not affect $e_{6}, e_{5}$, or $e_{4}$, so finally I have produced a basis with the desired properties.

Proposition 5.8. Let $\widetilde{\mathbb{P}}$ be a surface resulting from the blowing-up of a 6-point base locus $\left(P_{1}, \ldots, P_{6}\right)$ in $\mathbb{P}^{2}$ which is a union of towers such that no four base points are on a line. Let $D$ be a divisor class on $\widetilde{\mathbb{P}}$ such that the intersection multiplicity of $D$ with any (-2)-curve is nonnegative. Then there exists a map $\widetilde{\mathbb{P}} \rightarrow \mathbb{P}^{2}$ which is again the blowing-up of a (possibly different) 6-point base locus $\left(P_{1}^{\prime}, \ldots, P_{6}^{\prime}\right)$ in $\mathbb{P}^{2}$ such that no four base points are on a line and such that with respect to the induced basis $\ell, e_{1}, \ldots, e_{6}, D=\left(a ; b_{1}, \ldots, b_{6}\right)$ with the properties: 
- If $P_{j}^{\prime}$ is infinitely near to $P_{i}^{\prime}$, then $i<j$;

- $b_{1} \geq b_{2} \geq \cdots \geq b_{6}$

- $a \geq b_{1}+b_{2}+b_{3}$.

Proof. If $P_{j}$ is infinitely near to $P_{i}$, the condition D. $E_{j} \geq 0$ forces $D . e_{i} \geq D \cdot e_{j}$. Therefore, any renumbering of the points such that the second condition is satisfied will automatically satisfy the first condition as well. With respect to this numbering, $P_{1}$ is ordinary, $P_{2}$ is either ordinary or infinitely near to $P_{1}$, and $P_{3}$ is ordinary or infinitely near to either $P_{1}$ or $P_{2}$. Thus $S^{\prime}=P_{1}+P_{2}+P_{3}$ is a union of towers and either $P_{1}, P_{2}, P_{3}$ are collinear or $P_{1}, P_{2}, P_{3}$ determine a Cremona transformation of $\mathbb{P}^{2}$.

In the first case, $F_{123}$ is a (-2)-curve and D. $F_{123}=a-b_{1}-b_{2}-b_{3} \geq 0$, so the last condition holds. Otherwise, the transformation $\tau_{123}$ corresponds to an actual Cremona transformation of $\mathbb{P}^{2}$, so we can proceed as in Lemma 5.7 to obtain the desired basis.

Definition 5.9. Following the notation in [O'Sullivan 1996], I will refer to the basis determined in Proposition 5.8 for the divisor $D$ as a preferred basis for $D$. The 7-tuple of integers representing the divisor class of $D$ with respect to a preferred basis for $D$ is called a preferred form for $D$.

Proposition 5.10. Let $D=\left(a ; b_{1}, \ldots, b_{6}\right)$ be a divisor class on a smooth cubic surface $X$ with respect to the basis $\ell, e_{1}, \ldots, e_{6}$ such that $b_{1} \geq b_{2} \geq \cdots \geq b_{6}$ and $a \geq b_{1}+b_{2}+b_{3}$. Then $D$ is effective if and only if $a \geq \max \left(0, b_{1}\right)$.

Proof. That $a \geq \max \left(0, b_{1}\right)$ is necessary is fairly clear: $a$ cannot be negative for an effective divisor, and $a<b_{1}$ would mean that there was a plane curve of degree $a$ with a point of multiplicity more than $a$, which is impossible.

To prove sufficiency, let $r=\max \left\{i: b_{i} \geq 0\right\}$ and write $D=D_{+}+D_{-}$, where $D_{+}=\left(a ; b_{1}, \ldots, b_{r}, 0, \ldots, 0\right)$ and $D_{-}=\left(0 ; 0,0, \ldots, b_{r+1}, \ldots, b_{6}\right) . D_{-}$is clearly effective, as it is the sum of exceptional curves. At this point, either $D_{+}$satisfies the hypotheses of the proposition or $b_{3}<0$ and $a-b_{1}-b_{2}=c<0$. In the latter case, $D_{+}$intersects $F_{12}$ with multiplicity $-c$, so it contains at least $c$ copies of $F_{12}$. Now, make the further decomposition $D_{+}=D_{+}^{\prime}+c F_{12}$ and now $D_{+}^{\prime}$ does satisfy the hypotheses of the proposition.

So it suffices to show that the proposition holds for $D$ with all $b_{i} \geq 0$. First dispensing with an easy case, if $a=b_{1}$, then the other $b_{i}$ are all 0 and $D$ is a multiple of the conic $(1 ; 1,0,0,0,0,0)$.

Therefore assume that $a>b_{1}$. By [Hartshorne 1977, V, Ex. 4.8], if a divisor on a smooth cubic intersects each of the 27 lines nonnegatively and has nonnegative self-intersection, then that divisor is linearly equivalent to an effective divisor. The intersection with the lines is immediate: Since all of the entries for $D$ are positive, 
$D . E_{i} \geq 0$ for each $i$. Also, D. $F_{i j} \geq D . F_{12}$ by the condition $b_{1} \geq b_{2} \geq \cdots \geq b_{6}$, and D.F $F_{12}=a-b_{1}-b_{2} \geq 0$ by $a \geq b_{1}+b_{2}+b_{3}, b_{3} \geq 0$. Finally, D.G $G_{j} \geq D . G_{6}$, again by $b_{1} \geq b_{2} \geq \cdots \geq b_{6}$, and since $a \geq b_{1}+b_{2}+b_{3}$, certainly $a \geq b_{4}+b_{5}$, so D. $G_{6}=2 a-b_{1}-b_{2}-b_{3}-b_{4}-b_{5} \geq 0$. $D^{2}>0$ is an immediate consequence of the following lemma, which is readily verified.

Lemma 5.11. Let $\left(a ; b_{1}, b_{2}, \ldots, b_{6}\right)$ be a 7-tuple of nonnegative integers such that $a \geq b_{1}+b_{2}+b_{3}, a>b_{1}$, and $b_{1} \geq b_{2} \geq \cdots \geq b_{6}$. Then $a^{2}>\sum b_{i}^{2}$.

The arithmetic genus of an effective divisor on the blown-up surface. Let $X$ be a smooth surface and $D$ an effective divisor on $X$. Recall that the Zariski decomposition $[1962, \S 7]$ of $D$ is the unique decomposition $D=D_{L}+D_{N}$ with $D_{L}$ effective such that

(1) $D_{L}=0$ or the intersection matrix for each connected component of the support of $D_{L}$ is negative-definite;

(2) $D_{N}$ is numerically effective;

(3) $D_{N} \cdot E=0$ for each prime divisor $E$ in the support of $D_{L}$.

The following is needed for Proposition 5.14, which characterizes the Zariski decomposition of an effective divisor on one of our blown-up surfaces.

Proposition 5.12. Let $X$ be a smooth projective surface over $k$. Let $\widetilde{X} \stackrel{\pi}{\rightarrow} X$ be the composition of blowings-up of a sequence of closed points $\left(P_{1}, \ldots, P_{n}\right)$. Let $D$ be an exceptionally supported divisor such that D. $E_{i} \leq 0$ for each component $E_{i}$ of the exceptional locus. Then $D$ is effective.

Proof. This is immediate from the following lemma about bilinear forms, which is not difficult to verify.

Lemma 5.13. Let ( , ) be a negative-definite bilinear form on a finitely generated free abelian group $G$ and let $x_{1}, \ldots, x_{n}$ be a basis of $G$ such that $\left(x_{i}, x_{j}\right) \geq 0$ for $i \neq j$. If $y=\sum s_{i} x_{i}$ such that $\left(y, x_{i}\right) \leq 0$ for each $i$, then all of the $s_{i}$ are nonnegative.

Proposition 5.14. Let $\widetilde{\mathbb{P}}$ be the surface obtained by blowing up a curvillinear base locus in $\mathbb{P}^{2}$ of length 6 , and let $D$ be an effective divisor on $\widetilde{\mathbb{P}}$ having nonnegative intersection with all (-2)-curves, with Zariski decomposition $D=D_{L}+D_{N}$. Then $D_{N}$ is effective. Further, if $D$ is in preferred form, this decomposition for $D$ has one of the following forms:

(1) If $a-b_{1}-b_{2} \geq 0$, then $D_{N}$ has type $\left(a, b_{1}, \ldots, b_{r-1}, 0, \ldots, 0\right)$ and $D_{L}$ has type $\left(0 ; 0, \ldots, 0, b_{r}, \ldots, b_{6}\right)$, where $r$ is such that $b_{r-1} \geq 0$ and $b_{r}<0$.

(2) If $a-b_{1}-b_{2}=c<0$, then $D_{N}$ has type $\left(a+c ; b_{1}+c, b_{2}+c, 0,0,0,0\right)$ and $D_{L}$ has type $\left(-c ;-c,-c, b_{3}, \ldots, b_{6}\right)$ with $b_{3}, \ldots, b_{6}$ all less than 0 . 
Proof. First, we need to show that our purported $D_{L}$ is effective. This is immediate, because in each case $D_{L}$ is a positive linear combination of $e_{i}$ and $f_{12}=$ $(1 ; 1,1,0,0,0,0)$, which are always effective regardless of the collinearity or infinitely near behavior of the six points in $\mathbb{P}^{2}$. Further, in both cases each of these components is either a single $(-1)$-curve or a cluster of $(-2)$-curves together with a $(-1)$-curve all collapsing to a point in $\mathbb{P}^{2}$, and one can verify that its intersection matrix must be negative-definite.

To show that our $D_{N}$ is numerically effective, it suffices to show that $D_{N} \cdot E \geq$ 0 for any curve $E$ with negative self-intersection. For the exceptional curves $E_{1}, \ldots, E_{6}$, this follows from preferred form 5.8: If $E_{i}$ is a $(-1)$-curve, then $D_{N} \cdot E_{i}$ is just the $i$-th coefficient after the semicolon in the divisor type for $D_{N}$, which is always nonnegative. If $E_{i}$ is a (-2)-curve, say with $P_{j}$ infinitely near to $P_{i}$, then $i<j$; by the decreasing nature of the $b_{i}$ (which property is preserved when passing to $D_{L}$ ) the intersection number $D_{L} \cdot E_{i}=D_{L} \cdot e_{i}-D_{L} \cdot e_{j}$ remains nonnegative. Since $D$ was given in preferred form, it is easy to see that $D_{N} . F_{i j}$ is at least as large as $D_{N} \cdot F_{12}$.

To show that $D_{N}$ has 0 intersection with each component of $D_{L}$, note that, in case $1, D_{L}$ is supported on $E_{r}, \ldots, E_{6}$ and the result follows trivially from the intersection theory on $\widetilde{\mathbb{P}}$. For case $2, D_{L}$ is supported on $F_{12}$ or $F_{123}$, depending on whether $P_{1}, P_{2}, P_{3}$ are collinear, together with $E_{3}, \ldots, E_{6}$. By construction $D_{L} \cdot F_{12}=0$ or $D_{L} \cdot F_{123}=0$, and as in case $1 D_{L} \cdot E_{i}=0, i=3,4,5,6$.

Therefore, the Zariski decomposition is as stated. Effectiveness of $D_{N}$ now follows immediately from Proposition 5.12, since item (3) in the Zariski decomposition shows that $D_{N}$ is effective on the support of $D_{L}$, and away from this support it is effective because the original $D$ is.

Lemma 5.15. Let $\widetilde{\mathbb{P}}$ be the surface obtained from $\mathbb{P}^{2}$ by the sequential blowing-up of a curvillinear 6-point base locus with no four base points collinear. Let $|D|$ be a numerically effective divisor class on $\widetilde{\mathbb{P}}$. Then the general member of $|D|$ is a reduced divisor each of whose integral components has negative intersection with the canonical divisor $K_{\widetilde{\mathbb{P}}}$ of $\widetilde{\mathbb{P}}$.

Proof. Since $|D|$ is numerically effective, it has no fixed components by Proposition 5.14. Since there are only finitely many curves with negative self-intersection on $\widetilde{\mathbb{P}}$ by Proposition 2.3 , the general member of $|D|$ is supported on integral curves of nonnegative self-intersection. Such curves always move in linear equivalence classes, so the general member of $|D|$ is reduced. As $-K_{\widetilde{\mathbb{P}}}$ is very ample away from (-2)-curves, each component of the general member of $|D|$ has positive intersection with $-K_{\widetilde{\mathbb{P}}}$.

Proposition 5.16. Let $\widetilde{\mathbb{P}}$ be the surface obtained by the sequential blowing-up of a 6-point base locus in $\mathbb{P}^{2}$ which is a union of towers with no four base points 
collinear. Let $D \subset \widetilde{\mathbb{P}}$ be an effective divisor such that its intersection with each $(-2)$-curve is nonnegative. Write the divisor class of $D$ as $\left(a ; b_{1}, \ldots, b_{6}\right)$ with $b_{1} \geq b_{2} \geq \cdots \geq b_{6}$ and $a \geq b_{1}+b_{2}+b_{3}$ as in Proposition 5.8. Then

$$
h^{0} \mathrm{O}_{\widetilde{\mathbb{P}}}(D)=\left(\begin{array}{c}
a+2 \\
2
\end{array}\right)-\sum\left(\begin{array}{c}
b_{i}+1 \\
2
\end{array}\right)+\left(\begin{array}{c}
-c \\
2
\end{array}\right) \text {, }
$$

where $c=a-b_{1}-b_{2}$.

Proof. Write $D=D_{N}+D_{L}$ as in Proposition 5.14, where $D_{N}$ is numerically effective and effective and $D_{L}$ is common to all divisors linearly equivalent to $D$. Then $h^{0} \widetilde{O}_{\widetilde{\mathbb{P}}}(D)=h^{0} \widehat{O}_{\widetilde{\mathbb{P}}}\left(D_{N}\right)$. I claim that the formula also gives the same result for $D$ and $D_{N}$. To see this, first write the divisor type of $D_{N}$ as $\left(a_{N} ; b_{1 N}, \ldots, b_{6 N}\right)$.

If $c \geq 0$, then by Proposition 5.14, $a_{N}=a$ and $b_{i N} \leq b_{i}$ so that $c_{N}=a_{N}-b_{1 N}-$ $b_{2 N} \geq 0$ as well. Therefore,

$$
\left(\begin{array}{c}
-c \\
2
\end{array}\right)=0, \quad\left(\begin{array}{c}
-c_{N} \\
2
\end{array}\right)=0 .
$$

Also, the $b_{i}$ which contribute nonzero terms to the formula are exactly those which are greater than or equal to 1 , in which case $b_{i N}=b_{i}$ so the contributions are the same. If $b_{i}<1$, so is $b_{i N}$, so the contributions for these $i$ are both 0 .

If $c<0$, then $c_{N}=a-b_{1}-b_{2}-c=0$; furthermore, $b_{i}=b_{i N}=0$ for $i=3,4,5,6$. Therefore, for $D$ the formula gives

$$
\left(\begin{array}{c}
a+2 \\
2
\end{array}\right)-\left(\begin{array}{c}
b_{1}+1 \\
2
\end{array}\right)+\left(\begin{array}{c}
b_{2} \\
2
\end{array}\right)+\left(\begin{array}{c}
-c \\
2
\end{array}\right)
$$

note that in order for $c$ to be negative, $b_{1}$ and $b_{2}$ must both be positive. On the other hand, the formula for $D_{N}$ gives

$$
\left(\begin{array}{c}
a+c+2 \\
2
\end{array}\right)+\left(\begin{array}{c}
b_{1}+c+1 \\
2
\end{array}\right)+\left(\begin{array}{c}
b_{2}+c+1 \\
2
\end{array}\right)=\left(\begin{array}{c}
2 a-b_{1}-b_{2}+2 \\
2
\end{array}\right)+\left(\begin{array}{c}
a-b_{2} \\
2
\end{array}\right)+\left(\begin{array}{c}
a-b_{1} \\
2
\end{array}\right) .
$$

One can verify that each of the two sums evaluates to

$$
a^{2}+2 a-a b_{1}-a b_{2}+b_{1} b_{2}-b_{1}-b_{2}+1 .
$$

In either case, then, the formula gives the same result for $D$ and for $D_{N}$, as claimed.

It therefore suffices to prove the formula for $D_{N}$; for ease of notation, replace $D_{N}$ by $D$. Then $D$ is numerically effective, $c \geq 0$, and each $b_{i}$ is nonnegative by Proposition 5.14. Therefore, Lemma 5.15 shows that $D$ is linearly equivalent to a reduced divisor each of whose integral components has negative intersection with $K_{\widetilde{\mathbb{P}}}$; since we are proving a formula about dimensions of linear systems, we may as well assume that $D$ itself has this form. Consider the exact sequence on $D$

$$
0 \rightarrow O_{D} \rightarrow \mathrm{O}_{D}(D) \rightarrow \mathcal{T} \rightarrow 0,
$$


where $\mathscr{T}$ is a torsion sheaf of degree $D^{2}$. By Lemma 4.13, $H^{1}\left(\mathcal{O}_{D}(D)\right)=0$, so $H^{0}\left(\mathscr{O}_{D}(D)\right)=\chi\left(\mathscr{O}_{D}(D)\right)=\chi(\mathscr{T})+\chi\left(\mathscr{O}_{D}\right)=D^{2}+1-p_{a}(D)=\frac{1}{2}\left(D^{2}-D . K\right)$, the last equality coming from the adjunction formula. Now consider the exact sequence

$$
0 \rightarrow \mathrm{O}_{\widetilde{P}} \rightarrow \mathrm{O}_{\widetilde{\mathbb{P}}}(D) \rightarrow \mathrm{O}_{D}(D) \rightarrow 0
$$

and note that, since $\widetilde{\mathbb{P}}$ is rational, $H^{1}\left(\mathcal{O}_{\widetilde{\mathbb{P}}}\right)=0$. Therefore $H^{1}\left(\mathcal{O}_{\widetilde{\mathbb{P}}}(D)\right)=0$, and thus, using Proposition 2.2, we find that

$$
\begin{aligned}
H^{0}\left(\mathcal{O}_{\widetilde{\mathbb{P}}}(D)\right) & =\chi\left(\mathcal{O}_{\widetilde{\mathbb{P}}}(D)\right)=\chi\left(\mathcal{O}_{D}(D)\right)+\chi\left(\mathcal{O}_{\widetilde{\mathbb{P}}}\right) \\
& =\frac{1}{2}\left(D^{2}-D \cdot K\right)+1=\frac{1}{2}\left(\left(a^{2}-\sum b_{i}^{2}\right)+\left(3 a-\sum b_{i}\right)\right)+1 \\
& =\left(\begin{array}{c}
a+2 \\
2
\end{array}\right)-\sum\left(\begin{array}{c}
b_{i}+1 \\
2
\end{array}\right)+\left(\begin{array}{c}
-c \\
2
\end{array}\right) .
\end{aligned}
$$

Corollary 5.17. Let $\widetilde{\mathbb{P}}$ be a surface obtained by blowing up a 6-point base locus in $\mathbb{P}^{2}$ which is a union of towers with no four base points collinear. If an effective divisor $C$ on $\widetilde{\mathbb{P}}$ has nonnegative intersection with all-2-curves, then $C$ has the numerical type of an effective divisor on a smooth cubic surface.

Corollary 5.18. Let $\widetilde{\mathbb{P}}$ be the surface obtained from $\mathbb{P}^{2}$ by sequentially blowing up a curvillinear 6-point base locus with no four base points collinear. Let $D \subset \widetilde{\mathbb{P}}$ be an effective divisor such that its intersection with each (-2)-curve is nonnegative. Write the divisor class of $D$ as $\left(a ; b_{1}, \ldots, b_{6}\right)$ with $b_{1} \geq b_{2} \geq \cdots \geq b_{6}$ and $a \geq$ $b_{1}+b_{2}+b_{3}$ as in Proposition 5.8, and suppose further that $a \geq b_{1}+b_{2}$. Then $H^{1}\left(\widetilde{O}_{\widetilde{\mathbb{P}}}(D)\right)=0$.

Proof. The canonical sheaf of $\widetilde{\mathbb{P}}$ is antieffective, and $D$ is effective, so by Serre duality $H^{2}\left(\widetilde{O}_{\widetilde{\mathbb{P}}}(D)\right)=0$. Therefore, it suffices to show that $\chi\left(\mathcal{O}_{\widetilde{\mathbb{P}}}(D)\right)=H^{0}\left(\mathcal{O}_{\widetilde{\mathbb{P}}}(D)\right)$.

Consider the exact sequence

$$
0 \rightarrow \mathrm{O}_{D} \rightarrow \mathrm{O}_{D}(D) \rightarrow \mathscr{T} \rightarrow 0
$$

where $\mathscr{T}$ is a torsion sheaf of degree $D^{2}$. By definition, $\chi\left(O_{D}\right)=1-p_{a}(D)$; therefore,

$$
\chi\left(O_{D}(D)\right)=1-p_{a}(D)+D^{2} .
$$

Now use the exact sequence

$$
0 \rightarrow \mathrm{O}_{\widetilde{\mathbb{P}}} \rightarrow \mathrm{O}_{\widetilde{\mathbb{P}}}(D) \rightarrow \mathrm{O}_{D}(D) \rightarrow 0
$$

together with the fact that $H^{1}\left(\mathcal{O}_{\widetilde{\mathbb{P}}}\right)=H^{2}\left(\mathcal{O}_{\widetilde{\mathbb{P}}}\right)=0$ to see that

$$
\chi\left(\mathscr{O}_{\widetilde{\mathbb{P}}}(D)\right)=2-p_{a}(D)+D^{2} .
$$


Using Proposition 2.2, the right hand side can be expanded to

$$
2-\left(\begin{array}{c}
a-1 \\
2
\end{array}\right)+\sum\left(\begin{array}{c}
b_{i} \\
2
\end{array}\right)+a^{2}-\sum b_{i}^{2}
$$

which simplifies to

$$
\left(\begin{array}{c}
a+2 \\
2
\end{array}\right)-\sum\left(\begin{array}{c}
b_{i}+1 \\
2
\end{array}\right)
$$

Since by hypothesis $a \geq b_{1}+b_{2}$, this is equal to the formula for $H^{0}\left(\mathbb{O}_{\widetilde{\mathbb{P}}}(D)\right)$ found in Proposition 5.16.

Proposition 5.19. Let $T$ be a smooth connected (not necessarily complete) curve, and let $Z$ be a flat family of length- 6 schemes in $\mathbb{P}_{T}^{2}$ such that $Z_{t}$ is general for $t \neq 0$ and $Z_{0}$ is curvillinear meeting no line in multiplicity 4 . Let $X$ be the resulting family of blown-up surfaces. Let $D=\left(a ; b_{1}, \ldots, b_{6}\right) \in \operatorname{Pic} X / \mathrm{Pic} T$ as in Proposition 4.6 such that $D_{0}$ is effective and $D_{0} . E \geq 0$ for all $(-2)$-curves $E \subset X_{0}$. Then the dimensions of the cohomology groups $H^{0}\left(X_{t}, \mathrm{O}_{X_{t}}\left(D_{t}\right)\right)$ and $H^{1}\left(X_{t}, \mathrm{O}_{X_{t}}\left(D_{t}\right)\right)$ are constant over the family, and $H^{2}\left(X_{t}, O_{X_{t}}\left(D_{t}\right)\right)=0$ for all $t \in T$.

Proof. First, find a preferred basis for $D_{0}$ so that the integers $a, b_{1}, \ldots, b_{6}$ satisfy the conclusion of in Proposition 5.8. Now, $D_{0}$ satisfies the hypotheses of Proposition 5.16; therefore,

$$
h^{0} \mathrm{O}_{X_{0}}\left(D_{0}\right)=\left(\begin{array}{c}
a+2 \\
2
\end{array}\right)-\sum\left(\begin{array}{c}
b_{i}+1 \\
2
\end{array}\right)+\left(\begin{array}{c}
-c \\
2
\end{array}\right),
$$

where $c=a-b_{1}-b_{2}$. For this number to be positive, surely $a \geq b_{1}$, so by Proposition 5.10, $D_{t}$ is effective for all $t \in T$. Therefore, the formula equally well applies to all the $D_{t}$, so $H^{0}\left(X_{t}, O_{X_{t}}\left(D_{t}\right)\right)$ is constant on the family. Also, since the canonical divisor $K_{X_{t}}$ is antieffective, by Serre duality shows that $H^{2}\left(X_{t}, \mathcal{O}_{X_{t}}\left(D_{t}\right)\right) \perp$ $H^{0}\left(X_{t}, \mathrm{O}_{X_{t}}\left(K_{X_{t}}-D_{t}\right)\right)=0$. Now use the Riemann-Roch formula [Hartshorne 1977, V, Theorem 1.6] on the surface $X_{t}$ to show that

$$
h^{0}\left(X_{t}, \mathscr{O}_{X_{t}}\left(D_{t}\right)\right)-h^{1}\left(X_{t}, \mathcal{O}_{X_{t}}\left(D_{t}\right)\right)=\chi\left(\mathcal{O}_{X_{t}}\left(D_{t}\right)\right)=D_{t} \cdot\left(K_{X_{t}}-D_{t}\right) .
$$

Since this last intersection number depends only on the integers $a, b_{1}, \ldots, b_{6}$, and the $h^{0}$ term was already shown to be constant over the family, the $h^{1}$ must be constant over the family as well.

Proposition 5.20. Let $T$ be a smooth connected (not necessarily complete) curve, and let $Z$ be a flat family of length- 6 schemes in $\mathbb{P}_{T}^{2}$ such that $Z_{t}$ is general for $t \neq 0$ and $Z_{0}$ is curvillinear meeting no line in multiplicity 4 . Let $X$ be the resulting family of blown-up surfaces, and let $D_{0} \subset X_{0}$ be an effective divisor having nonnegative intersection with all (-2)-curves. Then, possibly after shrinking $T$, there exists an effective divisor $D$ on $X$ without vertical components whose restriction to $X_{0}$ is equal to $D_{0}$. 
Proof. By Proposition 5.19, the family satisfies the hypotheses of Proposition 4.12, which gives the desired result immediately.

The main theorem. At this point we have shown that any divisor on $X_{0}$ meeting certain conditions can be realized as the limit of a family of divisors on $X_{t}$. We will now show that for a given curve on a normal cubic surface, one of these families gives rise to a flat family specializing to the given curve.

The strategy involved will be to show that any curve on a normal cubic surface can be "linked up" to a smooth curve by adding hyperplane sections. Then we will compare genus formulas for our blown-up surfaces with known linkage formulas for surfaces in $\mathbb{P}^{3}$ and deduce that the special fibre of a suitably-chosen family from the blown-up surfaces will give a special fibre in $\mathbb{P}^{3}$ that has the right arithmetic genus to make a flat family. The following two propositions are thus needed to proceed.

Proposition 5.21. Let $\widetilde{\mathbb{P}}$ be a surface resulting from the blowing-up of a 6-point base locus in $\mathbb{P}^{2}$ which is a union of towers such that no four base points are on a line. Let $D$ be a divisor class on $\widetilde{\mathbb{P}}$ such that $D$ has small intersection with (-2)curves. Let $\mathcal{M}=-K=(3 ; 1,1,1,1,1,1)$ be the anticanonical divisor class of $\widetilde{\mathbb{P}}$. Then for $n$ sufficiently large, the divisor $D+n M$ is basepoint-free and gives a birational morphism to projective space whose image is a surface with isolated singularities.

Proof. First, by Proposition 5.8 we can find a preferred basis for $D$. Note that this basis is also preferred for $D+n M$, since the conditions for a basis to be preferred are unaffected by the addition or subtraction of $M$. By Proposition $2.4, \mathcal{M}$ is very ample away from $(-2)$-curves, so we may choose $n$ large enough that $D+n \mathcal{M}$ is also very ample away from (-2)-curves. By Lemma 5.15, we can also choose $n$ large enough that $D+n \mathcal{M}$ has no base components. Therefore, for such $n$ the general member of $|D+n M|$ is integral, since it is smooth away from (-2)-curves by Bertini's Theorem and has no $(-2)$-curves as components. If necessary add on another $\mathcal{M}$ so that $(D+n \mathcal{M}) . M>1$. I claim that for this value of $n, D^{\prime}=D+n \mathcal{M}$ is basepoint-free. It suffices to show that the sheaf $\mathcal{O}_{\widetilde{\mathbb{P}}}(D+n \mathcal{M})$ is generated by global sections at each point of the $(-2)$-curves.

As $D$ has small intersection with fundamental cycles, so does $D+n M$; therefore, for each $(-2)$-curve $E$ on $\widetilde{\mathbb{P}}$, the sheaf $O_{E}\left(D^{\prime}\right)$ is generated by global sections. Hence it will suffice to show that in the exact sequence

$$
0 \rightarrow \mathrm{O}_{\widetilde{\mathbb{P}}}\left(D^{\prime}-E\right) \rightarrow \mathrm{O}_{\widetilde{\mathbb{P}}}\left(D^{\prime}\right) \rightarrow \mathrm{O}_{E}\left(D^{\prime}\right) \rightarrow 0
$$

the map on the right is surjective on global sections; for this, in turn, it suffices to show that $H^{1}\left(\widetilde{O}_{\widetilde{\mathbb{P}}}\left(D^{\prime}-E\right)\right)=0$. 
Consider the exact sequence

$$
0 \rightarrow \mathrm{O}_{D^{\prime}}\left(D^{\prime}-E\right) \rightarrow \mathrm{O}_{D^{\prime}}\left(D^{\prime}\right) \rightarrow \mathrm{O}_{D^{\prime} \cap E}(D) \rightarrow 0 .
$$

Since $D^{\prime}$ has small intersection with fundamental cycles, $D^{\prime} \cap E$ is either empty or a reduced point, so the map on the right is surjective on global sections. On the other hand, by Lemma 4.13, $H^{1}\left(\mathscr{O}_{D^{\prime}}\left(D^{\prime}\right)\right)=0$, so this forces $H^{1}\left(\mathcal{O}_{\widetilde{\mathbb{P}}}\left(D^{\prime}-E\right)\right)=0$, as desired.

Therefore, the divisor $D+n \mathcal{M}$ is basepoint-free and thus determines a morphism from $\widetilde{\mathbb{P}}$ to projective space. It is an isomorphism away from (-2)-curves; therefore, the general hyperplane section is smooth away from the images of the $(-2)$-curves. Moreover, the general hyperplane section meets at most one (2)-curve in each cluster, and it is smooth there as well: In fact by Corollary 5.4 its image in the associated normal cubic surface remains smooth, so the general hyperplane section is smooth.

The particulars of the following formula are not important for our applications, only that this formula exists and depends only on the numerical data of the curves and surfaces involved.

Proposition 5.22 [Martin-Deschamps and Perrin 1990, Chapter III, Proposition 3.2]. Let $C$ be a curve of degree $d$ contained in the surface $Q$ of degree $s$ in $\mathbb{P}^{3}$. Let $H$ be the hyperplane class on $Q$, and let $D$ be a curve linearly equivalent to $C+n H$ for some integer $n$. Then

$$
p_{a}(D)=p_{a}(C)+n d+\frac{1}{2} n s(s+n-4) .
$$

Proposition 5.23. Let $X_{0} \subset \mathbb{P}^{3}$ be a normal cubic surface and $\widetilde{X}_{0} \stackrel{\pi}{\rightarrow} X_{0}$ its desingularization arising from the blowing-up of a curvillinear length-6 base locus with no four points on a line. Let $C_{0} \subset X_{0}$ be a (Cohen-Macaulay) curve. Then there exists a divisor $\bar{C}_{0}$ on $\tilde{X}_{0}$ such that

(a) $\left.\pi\right|_{\bar{C}_{0}}: \bar{C}_{0} \rightarrow C_{0}$ is an isomorphism away from (-2)-curves;

(b) $\bar{C}_{0}$ has small intersection with fundamental cycles;

(c) $p_{a}\left(\bar{C}_{0}\right)=p_{a}\left(C_{0}\right)$.

Proof. Let $\widetilde{C}_{0}$ be the strict transform of $C_{0}$ on $\widetilde{X}_{0}$. Then $\widetilde{C}_{0}$ has no $(-2)$-curves in its support, so its intersection with each $(-2)$-curve on $\widetilde{X}_{0}$ is nonnegative. Therefore by Proposition 5.5 , we can adjust $\widetilde{C}_{0}$ by an effective sum of $(-2)$-curves to obtain $\bar{C}_{0}$ having small intersection with fundamental cycles. This $\bar{C}_{0}$ satisfies items (a) and (b).

For item (c), let $\mu=-K$ be the divisor class $(3 ; 1,1,1,1,1,1)$ on $\widetilde{X}_{0}$. By Proposition 5.21, for large enough $n$ the linear system $\left|\mathscr{L}\left(\bar{C}_{0}\right)+n \mathcal{M}\right|$ is basepointfree and gives a birational map from $\widetilde{X}_{0}$ to a surface in projective space having 
isolated singularities. Fix such an $n$. Then the divisor class $\mathscr{L}\left(\bar{C}_{0}\right)+n \mu$ contains a smooth curve $\bar{D}_{0}$. As $M$ has 0 intersection with all (-2)-curves (remember that $\mathcal{M}$ gave the map $\pi$, which collapses all (-2)-curves), $\bar{D}_{0}$ still has small intersection with fundamental cycles. Therefore $\pi$ takes $\bar{D}_{0}$ isomorphically onto its image $D_{0}$ in $X_{0}$, so certainly $p_{a}\left(\bar{D}_{0}\right)=p_{a}\left(D_{0}\right)$. Also note that $D_{0}$ is linearly equivalent to $C_{0}+n H$ on $X_{0}$, where $H$ is the hyperplane class.

I claim that this forces $p_{a}\left(\bar{C}_{0}\right)=p_{a}\left(C_{0}\right)$. To see this, note that by Corollary 5.17 there is a curve $C$ on a smooth cubic surface $S$ with the same numerical type as $\bar{C}_{0}$, since $\bar{C}_{0}$ has nonnegative intersection with (-2)-curves. The degree of $C$ in $\mathbb{P}^{3}$ is equal to the degree of $C_{0}$, since in each case this is computed by taking the intersection with $(3 ; 1,1,1,1,1,1)$. Further, $p_{a}(C)=p_{a}\left(\bar{C}_{0}\right)$, since the arithmetic genus is computed purely by the numerical type of a divisor on a $\widetilde{X}_{0}$ or on a smooth cubic. Let $D$ be a curve in the class of $C+n H$ on $S$. Again by intersection theory, $p_{a}(D)=p_{a}\left(\bar{D}_{0}\right)$, which is equal to $p_{a}\left(D_{0}\right)$ from the preceding paragraph, and also $D$ and $D_{0}$ have the same degree. Therefore, the formula in Proposition 5.22 shows that $p_{a}\left(C_{0}\right)=p_{a}(C)$, and thus that $p_{a}\left(C_{0}\right)=p_{a}\left(\bar{C}_{0}\right)$.

Theorem 5.24. Let $X_{0} \subset \mathbb{P}^{3}$ be the rational normal cubic surface associated to the blow-up $\widetilde{X_{0}}$ of $\mathbb{P}^{2}$ at a set $S_{0}$ of 6 points which is a union of towers with no four points collinear, corresponding to the subscheme $Z_{0}$ with complete ideal $\Phi_{Z_{0}}$. Let $Z \subset \mathbb{P}_{T}^{2}$ be a flat family over a (not necessarily complete) smooth curve $T$ with $0 \in T$ such that $Z_{t}$ is general for $t \neq 0$ and $(Z)_{0}=Z_{0}$. As in Proposition 4.11, this construction gives rise to a family $\widetilde{X}$ of smooth surfaces which maps into $\mathbb{P}_{T}^{3}$ as a family $X$ of normal cubic surfaces with general member smooth and with special member $X_{0}$. Then (possibly after shrinking $T$ ) any effective Weil divisor on $X_{0}$ is the limit of a flat family of curves on $X_{t}$.

Proof. Let $D_{0} \subset X_{0}$ be any effective Weil divisor. Let $\widetilde{D}_{0}$ be its strict transform on $\widetilde{X}_{0}$ (that is, the sum of the appropriate multiples of the strict transforms of the irreducible components of $D_{0}$ ). By the last proposition, there exists a divisor $\bar{D}_{0}$ on $\widetilde{X}_{0}$ which has small intersection with fundamental cycles, which has the same arithmetic genus as $C_{0}$, and such that $\left.\pi\right|_{\bar{D}_{0}}: \bar{D}_{0} \rightarrow D_{0}$ is generically an isomorphism. Then by Proposition 5.20, $\bar{D}_{0}$ is the limit of a flat family of divisors on $X$. The image of this family under $\pi$ is a family of curves parametrized by T. By [Hartshorne 1977, Theorem III.9.7], this image is a flat family it suffices to check Hilbert polynomials. The degree of $D_{0}$ is equal to the degrees of each of the $D_{t}$ by the intersection properties on the smooth surfaces $X_{t}$. The arithmetic genus is constant since $p_{a}\left(D_{0}\right)=p_{a}\left(\bar{D}_{0}\right)$. Therefore the Hilbert polynomials are constant over the family, and $D_{0}$ has been expressed as the limit of a flat family, as desired. 


\section{Acknowledgments}

Special thanks to Mike O'Sullivan: much of this work arose out of our very fruitful collaboration. Thanks also to Robin Hartshorne, for his guidance, mentorship, and countless suggestions; and to Rich Liebling, Scott Nollett, and Enrico Schlesinger, for many stimulating discussions.

This work was carried out while I was at the Department of Computer Science, University of California, Santa Barbara.

\section{References}

[Artin 1962] M. Artin, "Some numerical criteria for contractability of curves on algebraic surfaces", Amer. J. Math. 84 (1962), 485-496. MR 26 \#3704 Zbl 0105.14404

[Artin 1966] M. Artin, “On isolated rational singularities of surfaces”, Amer. J. Math. 88 (1966), 129-136. MR 33 \#7340 Zbl 0142.18602

[Balcerzyk and Józefiak 1989] S. Balcerzyk and T. Józefiak, Commutative rings: dimension, multiplicity and homological methods, Ellis Horwood, Chichester, UK, 1989. MR 92b:13001 Zbl 0685.13002

[Brevik and Mordasini 2003] J. Brevik and F. Mordasini, "Curves on a ruled cubic surface", Collect. Math. 54:3 (2003), 269-281. MR 2005c:14041 Zbl 1051.14037

[Bruce and Wall 1979] J. W. Bruce and C. T. C. Wall, "On the classification of cubic surfaces", J. London Math. Soc. (2) 19:2 (1979), 245-256. MR 80f:14021 Zbl 0393.14007

[Fogarty 1968] J. Fogarty, "Algebraic families on an algebraic surface", Amer. J. Math 90 (1968), 511-521. MR 38 \#5778 Zbl 0176.18401

[Gruson and Peskine 1982] L. Gruson and C. Peskine, "Genre des courbes de l'espace projectif. II", Ann. Sci. École Norm. Sup. (4) 15:3 (1982), 401-418. MR 84j:14034 Zbl 0517.14007

[Hartshorne 1977] R. Hartshorne, Algebraic geometry, Graduate Texts in Mathematics 52, Springer, New York, 1977. MR 57 \#3116 Zbl 0367.14001

[Hartshorne 1980] R. Hartshorne, "On the classification of algebraic space curves", pp. 83-112 in Vector bundles and differential equations (Nice, 1979), edited by A. Hirschowitz, Progr. Math. 7 , Birkhäuser, Boston, 1980. MR 81m:14022 Zbl 0452.14005

[Hartshorne 1997] R. Hartshorne, Families of curves in $\mathbf{P}^{3}$ and Zeuthen's problem, Mem. Amer. Math. Soc. 617, Amer. Math. Soc., Providence, RI, 1997. MR 98f:14024 Zbl 0894.14001

[Lipman 1969] J. Lipman, "Rational singularities, with applications to algebraic surfaces and unique factorization”, Inst. Hautes Études Sci. Publ. Math. 36 (1969), 195-279. MR 43 \#1986 Zbl 0181. 48903

[Lipman 1988] J. Lipman, "On complete ideals in regular local rings", pp. 203-231 in Algebraic geometry and commutative algebra, vol. I, edited by H. Hijikata et al., Kinokuniya, Tokyo, and Academic Press, Orlando, 1988. MR 90g:14003 Zbl 0693.13011

[Martin-Deschamps and Perrin 1990] M. Martin-Deschamps and D. Perrin, Sur la classification des courbes gauches, Astérisque 184-185, Soc. mathématique de France, Paris, 1990. MR 91h:14039 Zbl 0717.14017

[Mori 1984] S. Mori, "On degrees and genera of curves on smooth quartic surfaces in $\mathbf{P}^{3}$ ", Nagoya Math. J. 96 (1984), 127-132. MR 86g:14023 Zbl 0576.14032 
[Nagata 1960] M. Nagata, "On rational surfaces, I: Irreducible curves of arithmetic genus 0 or 1", Mem. Coll. Sci. Univ. Kyoto Ser. A Math. 33 (1960), 351-370. MR 23 \#A3740 Zbl 0100.16703

[O'Sullivan 1996] M. O'Sullivan, Normal cubic surfaces in $\mathbb{P}^{3}$, Ph.D. thesis, University of California, Berkeley, 1996.

[Zariski 1962] O. Zariski, "The theorem of Riemann-Roch for high multiples of an effective divisor on an algebraic surface", Ann. of Math. (2) 76 (1962), 560-615. MR 25 \#5065 Zbl 0124.37001

[Zariski 1971] O. Zariski, Algebraic surfaces, 2nd ed., Ergebnisse der Mathematik 61, Springer, New York, 1971. MR 57 \#9695 Zbl 0219.14020

[Zariski and Samuel 1960] O. Zariski and P. Samuel, Commutative algebra, vol. II, Van Nostrand, Princeton, NJ, 1960. MR 22 \#11006 Zbl 0121.27801

Received August 19, 2005. Revised January 9, 2006.

JOHN BREVIK

Department of MATHEMATiCs AND Statistics

1250 BELLFLOWER BOULEVARD

CALIFORNIA STATE UNIVERSITY

LONG BEACH, CA 90840-1001

United STATES

jbrevik@csulb.edu 